\title{
Le sentiment de discrimination des représentants du personnel
}

Une étude à partir des données statistiques et monographiques liées à l'enquête REPONSE

The Feeling of Discrimination among Trade Unionists. A Study Based on the Data from the French Survey REPONSE and Three Monographs

Baptiste Giraud, Amaël Marchand et Étienne Penissat

\section{OpenEdition}

Journals

\section{Édition électronique}

URL : http://journals.openedition.org/travailemploi/6908

DOI : 10.4000/travailemploi.6908

ISSN : 1775-416X

Éditeur

DARES - Ministère du Travail

\section{Édition imprimée}

Date de publication : 1 janvier 2016

Pagination : 87-119

ISSN : 0224-4365

\section{Référence électronique}

Baptiste Giraud, Amaël Marchand et Étienne Penissat, « Le sentiment de discrimination des

représentants du personnel », Travail et Emploi [En ligne], 145 | janvier-mars 2016, mis en ligne le 01 janvier 2018, consulté le 01 mai 2019. URL : http://journals.openedition.org/travailemploi/6908 ; DOI : 10.4000/travailemploi.6908 


\title{
Le sentiment de discrimination des représentants du personnel
}

\section{Une étude à partir des données statistiques et monographiques liées à l'enquête REPONSE}

\author{
Baptiste Giraud", Amaël Marchand ${ }^{* * *}$, Étienne Penissat ${ }^{* * * *}$
}

\begin{abstract}
Alors que les travaux sur la discrimination syndicale se sont essentiellement intéressés à l'objectivation des pénalités salariales subies par les syndicalistes, que ce soit devant les tribunaux ou dans les données statistiques, cet article s'intéresse aux ressorts sociaux du sentiment de discrimination. En s'appuyant sur les données de l'enquête Relations professionnelles et négociations d'entreprise (REPONSE) et sur trois monographies, il analyse les conditions qui font varier cette perception des risques liés à l'engagement syndical, en montrant notamment qu'il est plus prononcé dans les grands établissements, là où les relations professionnelles sont les plus institutionnalisées. La perception de cette discrimination s' active ainsi au croisement de quatre types de rapports sociaux : le rapport des syndicalistes aux autres salariés ; leur rapport aux directions d'entreprise, marqué par l'état des relations sociales et rapports de force avec les dirigeants ; leur rapport au droit et, plus largement, à la notion de discrimination ; et, enfin, le rapport des syndicalistes entre eux, par l'intermédiaire des pratiques distinctives entre syndicats et/ou syndicalistes selon leurs positions et leurs trajectoires militantes.
\end{abstract}

Tes travaux français sur les discriminations sont dominés par deux grandes Lapproches : ceux qui portent sur la construction de la « cause des discriminations » et sa reconnaissance institutionnelle et ceux qui visent à objectiver statistiquement

\footnotetext{
* Cet article découle d'une post-enquête financée par la Direction de l'animation de la recherche, des études et des statistiques (Dares) en convention avec le laboratoire Professions, institutions, temporalités (Printemps, université de Versailles Saint-Quentin-en-Yvelines, CNRS). Convention n ${ }^{\circ} 2200590601$, « La dialectique des conflits et des négociations en entreprise ».

** Aix-Marseille Université, CNRS, Lest, Aix-en-Provence, France ; baptiste.giraud@univ-amu.fr.

*** Laboratoire technique territoire et sociétés (Latts) ; amael.marchand@enpc.fr.

**** Centre d'études et de recherches administratives, politiques et sociales ; etienne.penissat@ univ-lille2.fr.
} 
les traitements inégaux dont sont victimes certains groupes sociaux, le plus souvent relativement à leur sexe ou à leur appartenance ethnique, par rapport aux groupes dominants. C'est également le cas concernant la discrimination syndicale - entendue comme un traitement spécifique et désavantageux de salariés en raison de leur appartenance syndicale - dont le traitement s'est d'abord construit à partir d'un travail d'objectivation des pénalités subies par les syndicalistes dans la progression de leur carrière et/ou de leur salaire du fait de leur engagement. Ainsi, des études statistiques et économétriques (BouRdieu, BREDA, dans ce numéro ; BREDA, 2014) reposant sur la neutralisation d'une série de variables relatives aux caractéristiques sociales et professionnelles (sexe, âge, ancienneté, qualification, temps passé dans le mandat) du représentant du personnel ont montré qu'en dernier ressort c'est bien l'engagement syndical qui «explique » la pénalisation. Cette méthode est d'ailleurs celle reconnue par les tribunaux pour apporter la preuve de ces discriminations (CHAPPE, 2013). Du côté de la «cause » de la discrimination syndicale, Vincent-Arnaud CHAPPE (2013) a mis en évidence que l'utilisation de la catégorie de discrimination était le fruit d'un contexte syndical particulier et que sa diffusion était le produit d'un travail de construction et de transmission de dispositifs matériels et cognitifs permettant une réappropriation par les syndicalistes.

En revanche, comme pour les autres formes de discriminations (PRIMON, 2011 ; ECKERT, PRIMON, 2011), les travaux qui cherchent à questionner la perception ou l'expérience des discriminations syndicales sont moins nombreux ${ }^{1}$. Des données statistiques permettent toutefois de commencer à les appréhender et à en analyser les conditions de production. Le baromètre du Défenseur des droits et de l'Organisation internationale du travail sur les discriminations au travail souligne ainsi combien l'appartenance syndicale est perçue comme un facteur de discrimination par les salariés (OBSERVATOIRE DE LA RÉPRESSION ET DE LA DISCRIMINATION SYNDICALES, 2014). Par ailleurs, d'après la dernière enquête Relations professionnelles et négociations d'entreprise (REPONSE), un tiers des représentants du personnel syndiqués déclarent que leur fonction a été un frein à leur carrière professionnelle (PAK, PIGNONI, 2014).

Or, s'intéresser à ces déclarations, c'est-à-dire au sentiment de discrimination, permet de porter un regard différent sur les enjeux sociaux et politiques. En effet, ce sentiment ne suppose pas nécessairement que soit attestée une discrimination « objective », telle que définie par le droit et repérée dans les études économétriques. À l'inverse, tous les syndicalistes dont on pourrait prouver qu' ils subissent des formes objectives de discrimination ne reprennent ou n'adhèrent pas à cette grille de lecture de leur situation. Elle fait d'ailleurs débat parmi les syndicalistes, comme l'illustre, dans

1. L'enquête Trajectoires et origines (TEO) de l'Institut national de la statistique et des études économiques (Insee) et de l'Institut national d'études démographiques (Ined) constitue une exception puisqu'elle comporte une question demandant aux enquêtés : «Est-il déjà arrivé que l'on se moque de vous, que l'on vous mette à l'écart, que l'on vous traite de façon injuste ou que l'on vous refuse un droit à cause de votre appartenance à une organisation politique ou syndicale » et une autre à cause de « vos opinions politiques, syndicales ou religieuses ? La formulation de la question ne permet toutefois pas d'isoler le sentiment de discrimination syndicale. Pour une présentation des résultats, lire Algava, BèQue, 2006. 
son échange avec le sociologue Michel Pialoux, le témoignage de l'ouvrier spécialisé cégétiste de Peugeot, Christian Corouge. Ce dernier, considérant son activité syndicale comme l'une de ses principales rétributions symboliques dans l'usine et la discrimination syndicale comme une contrepartie logique à cet investissement militant contre ses patrons, a toujours refusé de mener une action juridique pour faire reconnaître sa discrimination syndicale (Corouge, Pialoux, 2011). Les dimensions juridiques et subjectives du phénomène de discrimination syndicale ne se recouvrent donc pas totalement.

L'intérêt d'une entrée par le sentiment de discrimination est double. D'abord, alors que les approches habituelles se focalisent sur la discrimination telle qu'elle est définie par les entrepreneurs de la cause, par le droit ou par les chercheurs, recentrer l'attention sur les déclarations des représentants du personnel permet de mieux saisir ce que recouvre pour eux la notion de discrimination. On peut ainsi être plus attentif à la manière dont ils perçoivent les conséquences de leur engagement, dont ils qualifient leurs relations avec leur direction et aux conditions dans lesquelles ils peuvent se dire discriminés. Les situations de discrimination les plus souvent codifiées et reconnues par la loi (pénalité salariale, frein à la carrière professionnelle) n'épuisent pas la diversité des motifs pour lesquels les représentants du personnel peuvent éprouver le sentiment d'être pénalisés ou réprimés du fait de leur engagement syndical. Se limiter à l'étude des situations de discrimination syndicale sanctionnées par la loi apparaît insuffisant si l'on veut comprendre le maintien d'un sentiment de discrimination diffus chez les militants syndicaux, dans un contexte de codification juridique croissante des relations sociales, qui implique une transformation des stratégies managériales de canalisation du syndicalisme (GIRAUD, 2013). Par ailleurs, une approche plus compréhensive de la discrimination syndicale, tel qu'elle est perçue par les représentants du personnel, invite à étudier les situations de manière moins figée, pour repérer comment se construisent l'expérience et la perception des discriminations dans une perspective plus relationnelle (TILLY, 1998) et interactionniste (SCHWAlBE et al., 2000). Enfin, l'analyse des déclarations des représentants du personnel conduit à ne pas les appréhender comme un groupe homogène, mais à repérer et à analyser au contraire les différences entre syndicalistes dans le rapport à ce qui peut être qualifié de discrimination. Il s'agit donc moins de savoir si les représentants du personnel sont globalement traités de manière inégalitaire que de comprendre selon quelles logiques se construit leur propension différente à se sentir discriminés à cause de leur activité de représentant.

Pour contribuer à l'analyse des ressorts du sentiment de discrimination exprimé par les représentants du personnel, nous allons nous appuyer sur l'exploitation des données statistiques de l'enquête REPONSE, 2011, mise en perspective avec trois monographies d'entreprise réalisées dans le cadre d'une post-enquête collective (GIRAUD et al., 2014). Cette enquête est particulièrement adaptée à ce programme de recherche (voir encadré). Outre qu'elle permet d'objectiver la pénalisation salariale subie par les syndicalistes (BREDA, 2014 ; BouRDIEU, BREDA, dans ce numéro), elle donne la possibilité de saisir la perception qu'ils ont des désavantages qu'ils subissent 
en raison de leur mandat. Même si l'enquête ne comporte pas de questions mobilisant directement la notion de discrimination, elle permet de repérer les configurations d'établissement dans lesquelles les représentants de la liste majoritaire perçoivent leur fonction comme un risque pour leur emploi et un frein dans leur carrière professionnelle. En complément, les trois études de cas monographiques permettent d'affiner la compréhension du sentiment de discrimination et de mettre en débat certaines des limites de l'enquête statistique. Elles aident en particulier à mieux rendre compte de la diversité des motifs qui justifient que des syndicalistes s'estiment discriminés, et de celle des conditions sociales qui facilitent l'expression de ce sentiment.

Dans cette optique, nous verrons que l'un des principaux apports de l'enquête REPONSE est de mettre en évidence que le sentiment de discrimination s'exprime d'abord dans les grands établissements, dans lesquels la présence syndicale et les pratiques de la négociation collective sont les plus institutionnalisées, les représentants plus actifs et les conflits collectifs plus fréquents. Puis nous montrerons que, dans ces configurations d'établissement, le sentiment de discrimination syndicale qui s'exprime est pluridimensionnel, variable et relationnel : il est associé au sentiment plus collectif d'être entravé dans l'exercice de ses droits syndicaux, dépend de la manière dont les représentants s'engagent dans leur mandat et se construit aussi par le jeu de la comparaison qui s'établit entre eux. Pour conclure, on s'intéressera au processus de socialisation au droit des représentants, envisagé comme l'un des facteurs qui conditionnent l'usage de la notion de discrimination en situation de conflit avec la direction.

\section{ENCADRÉ}

\section{L'enquête REPONSE, la post-enquête et le sentiment de discrimination syndicale}

L'enquête Relations professionnelles et négociations d'entreprise (REPONSE) a lieu tous les six ans depuis 1993. La dernière édition date de 2011. Menée auprès d'environ 4000 établissements d'au moins onze salariés du secteur marchand non agricole, elle est composée de trois volets : un questionnaire conduit en face-à-face auprès des représentants des directions ( $R D, n=4023)$; un questionnaire en face-à-face auprès du représentant du personnel de la liste majoritaire, lorsqu'il en existe un et que le RD a donné son accord ( $R P, n=2433)$; un questionnaire postal auprès de salariés tirés au sort parmi l'ensemble des salariés des établissements sélectionnés pour l'enquête $(\mathrm{n}=18$ 536).

Au-delà des différentes caractéristiques des établissements, le questionnement porte, entre autres thèmes, sur les politiques de gestion du personnel et les politiques salariales, la présence et la nature des instances de représentation du personnel, la tenue de négociation et la conclusion d'accords collectifs, l'existence de conflits collectifs et la perception du climat social. Dans le cadre de cet article, nous avons plus spécifiquement utilisé deux questions du volet RP qui permettent d'appréhender la manière dont les représentants du personnel se sentent (ou non) pénalisés par l'exercice de leur mandat :

a) Concernant la stabilité de votre emploi, votre situation de représentant du personnel a-t-elle été : une protection / une menace / ni l'un, ni l'autre / (ne sait pas) ? 
b) Concernant votre évolution professionnelle au sein de l'entreprise, votre expérience de représentant du personnel a-t-elle été : un moteur / un frein / ni l'un, ni l'autre / (ne sait pas)?

Comme il ne recueille que l'avis du représentant de la liste majoritaire, ce questionnaire ne permet d'approcher le phénomène de la discrimination syndicale qu'à partir de son point de vue (nécessairement subjectif et situé) et de questions indirectes, qui ne font pas explicitement référence ni au terme de discrimination, ni à certaines situations de discrimination interdites par la loi : sanctions injustifiées, refus d'embauche, différences de traitement dans l'exercice du mandat, etc.

La mise en perspective des résultats de l'enquête statistique avec les données d'observation tirées de trois enquêtes monographiques permet de compenser en partie cette lacune, en nous donnant précisément les moyens de saisir la diversité des motifs pour lesquels des représentants se disent pénalisés quand ils déclarent que leur mandat constitue un frein pour leur carrière professionnelle ou un risque pour leur maintien dans l'emploi.

Les établissements étudiés ont été initialement choisis dans le cadre d'une postenquête REPONSE portant sur la dynamique des relations professionnelles en entreprise (cf. GIRAUD et al., 2014, pour une présentation plus détaillée de ces quatre études de cas). Dans notre questionnement initial, la problématique de la discrimination syndicale n'était donc pas présente en tant qu'objet d'enquête autonome. Lors de notre terrain, elle est cependant ressortie à plusieurs reprises au cours des entretiens menés avec les représentants. Nous l'avons dès lors approfondie : dans ces établissements, elle est apparue dans des contextes institutionnels sur lesquels il nous est paru particulièrement opportun de revenir au regard des questions que soulèvent les résultats de l'enquête statistique.

Les trois établissements dont il sera ici question, que l'on nommera Engins (280 salariés, majorité d'ouvriers qualifiés et non qualifiés), Transformateur (430, majorité de techniciens et ouvriers qualifiés) et Bus (250, majorité de chauffeurs) appartiennent à des grands groupes étrangers ou français. En cela, ils permettent de s'interroger sur l'existence d'un sentiment de discrimination là où plusieurs syndicats coexistent et où les relations sociales sont institutionnalisées. Les deux premiers sont des établissements industriels avec une présence syndicale réelle (environ $15 \%$ de syndiqués) et ancienne, la Confédération générale du travail (CGT) étant le syndicat majoritaire principalement implanté dans le collège ouvrier. Ils ont connu de nombreuses restructurations et plans sociaux dans les années 2000. Le troisième relève du secteur des services (transports publics), dans lequel on trouve trois sections syndicales, SUD étant majoritaire. Dans les deux premiers, la direction cherche à faire émerger une représentation syndicale plus coopérative tandis que chez Bus, la gestion des syndicats est conflictuelle. En tout, vingt-huit entretiens ont été conduits, principalement avec les syndicalistes de ces établissements, mais aussi avec les cadres locaux de direction.

\section{Les ressorts organisationnels du sentiment de discrimination}

À la différence de la discrimination raciale ou sexuée, dont l'expérience peut être éprouvée sur plusieurs scènes de la vie sociale (scolarité, travail, citoyenneté, loisirs, etc.) - ce qui amène d'ailleurs à parler d' « expérience totale » pour ceux qui en sont 
victimes (DUBET et al., 2013) -, la discrimination syndicale reste essentiellement concentrée dans l'univers professionnel. D'où la nécessité de comprendre les contextes organisationnels qui activent sa perception et sa reconnaissance par les syndicalistes. On montre ainsi que ce sentiment de discrimination est inégalement partagé par les représentants du personnel et qu'il dépend à la fois du type de mandat exercé et des caractéristiques des établissements.

\section{Un sentiment de discrimination propre aux syndicalistes}

L'appartenance à un syndicat fonctionne comme le principal facteur différenciant les représentants qui considèrent que leur mandat freine leur carrière et ceux qui estiment qu'il est un moteur ou qu'il ne joue pas sur leur développement de carrière (voir tableau 1). Dans les établissements où il y a des représentants du personnel élus et où la liste majoritaire est syndicale, les représentants appartenant à cette liste sont $25 \%$ à déclarer que leur mandat a freiné leur évolution professionnelle. Ce taux est encore plus élevé (35\%) dans les établissements où le représentant de la liste majoritaire est délégué syndical (et non seulement élu comme délégué du personnel ou membre du comité d'entreprise). Ce sentiment de discrimination ${ }^{2}$ n'est en revanche évoqué que de façon résiduelle par les représentants non syndiqués dans les établissements où il y a des élus du personnel et où la liste majoritaire est non syndicale (4\%). Autrement dit, plus que l'exercice d'un mandat de représentation du personnel, c'est le fait d'être syndiqué qui est déterminant pour comprendre le sentiment de discrimination. Même si un tel sentiment n'est pas nécessairement lié à une situation de discrimination effective, ce résultat semble néanmoins converger avec les études économétriques qui montrent, à partir d'une mesure objective de la discrimination, que, parmi les représentants du personnel, seuls les délégués syndicaux subissent des pénalités salariales (BREDA, 2014 ; BouRdieu, BREDA, dans ce numéro) ${ }^{3}$.

La déclaration par les représentants d'une menace possible sur leur emploi en raison de l'exercice d'un mandat est beaucoup plus faible, quelle que soit la caractéristique de la liste majoritaire (voir tableau 2). Ceci peut s'expliquer par le fait que les représentants élus ou mandatés par leurs syndicats bénéficient d'une protection spécifique dans le Code du travail qui oblige les directions d'entreprise à obtenir l'accord de l'inspection du travail pour licencier, contrôle a priori qui n'existe pas en matière de progression de carrière ou d'augmentation salariale. Comme nous l'avons observé dans nos monographies, l'affiliation à un syndicat (et la prise d'un mandat) peut d'ailleurs apparaître aux yeux de certains représentants comme une protection pour leur

2. Par la suite, lorsque la distinction n'est pas précisée, les données statistiques concernant le « sentiment de discrimination » porteront sur la question concernant l'effet du mandat sur la carrière professionnelle.

3. On retrouverait ici le même type de convergence entre discrimination objective et subjective, que celle identifiée dans les enquêtes sociologiques sur les discriminations raciales (SILbERman, Fournier, 2006 ; BrinBaum, GuÉGNARD, 2012). De la même manière que pour les syndicalistes aux prises avec différentes formes d'entrave à leur action et leur carrière, ces travaux pointent le rôle de la répétition dans le temps et dans l'espace des expériences pratiques de stigmatisation et de discrimination dans la construction du sentiment de discrimination chez les jeunes immigrés. 
emploi, et envisagée comme une condition préalable nécessaire à la possibilité d'un engagement syndical. C'est par exemple le cas de M. Henri, délégué syndical CGT de l'établissement Transformateur, qui produit des transformateurs électriques pour de grandes entreprises comme EDF ou la $\mathrm{SNCF}^{5}$. Dans ses précédents emplois au sein de petites entreprises, il estime avoir subi une véritable répression patronale, répression allant jusqu'à un « licenciement camouflé parce que justement [il] faisai[t] partie de ceux qui allaient se mettre au-devant des problèmes des collègues ». M. Henri explique alors avoir fait le choix d'adhérer à la CGT et de solliciter un mandat d'élu dès son entrée à Transformateur, afin de ne pas risquer de se retrouver à nouveau exposé à ce type de représailles patronales.

\section{TABLEAU 1 - Concernant votre évolution professionnelle au sein de l'entreprise, votre expérience de représentant du personnel a-t-elle été...}

$\%$ d'établissements

\begin{tabular}{lccc}
\hline & Un moteur & Un frein & Ni l'un, ni l'autre \\
\hline Élus non syndiqués & 12 & 4 & 84 \\
Élus syndiqués* & 14 & 25 & 61 \\
Délégués syndicaux & 12 & 35 & 53 \\
\hline
\end{tabular}

* Élus du personnel qui sont syndiqués mais n'exercent pas de fonction de délégué syndical ${ }^{6}$.

Lecture : dans $25 \%$ des établissements où il y a des représentants du personnel élus et où la liste majoritaire est syndicale, le représentant interrogé qui appartient à cette liste déclare que son mandat a été un frein à sa carrière, contre $4 \%$ des établissements où il y a des représentants du personnel élus et où la liste majoritaire est non syndicale (le représentant interrogé appartenant à cette liste).

Champ : établissements d'au moins 11 salariés du secteur marchand non agricole.

Source : REPONSE, 2011, volet « représentants du personnel », Dares $(\mathrm{n}=2433)$.

\section{TABLEAU 2 - Concernant la stabilité de votre emploi, votre situation de représentant du personnel a-t-elle été...}

$\%$ d'établissements

\begin{tabular}{lccc}
\hline & Une protection & Une menace & Ni l'un, ni l'autre \\
\hline Élus non syndiqués & 7 & 2 & 91 \\
Élus syndiqués* & 11 & 12 & 77 \\
Délégués syndicaux & 11 & 13 & 76 \\
\hline
\end{tabular}

* Élus du personnel qui sont syndiqués mais n'exercent pas de fonction de délégué syndical.

Lecture : dans $12 \%$ des établissements où il y a des représentants du personnel élus et où la liste majoritaire est syndicale, le représentant du personnel interrogé qui appartient à cette liste déclare que son mandat est une menace pour la stabilité de son emploi contre $2 \%$ des établissements où il y a des représentants du personnel élus et où la liste majoritaire est non syndicale (le représentant du personnel interrogé appartenant à cette liste).

Champ : établissements d'au moins 11 salariés du secteur marchand non agricole.

Source : REPONSE, 2011, volet « représentants du personnel », Dares $(\mathrm{n}=2433)$.

4. Confédération générale du travail.

5. EDF : Électricité de France ; SNCF : Société nationale des chemins de fer français.

6. Depuis la loi n ${ }^{\circ} 2008-789$ portant rénovation de la démocratie sociale et réforme du temps de travail, les délégués syndicaux, dans les entreprises de 50 salariés et plus, sont désignés parmi les candidats aux élections professionnelles. «Ils doivent avoir recueilli, à titre personnel et dans leur collège, au moins $10 \%$ des suffrages exprimés au premier tour des dernières élections au comité d'entreprise ou de la délégation unique du personnel ou des délégués du personnel. » (Source : https://www.service-public.fr/particuliers/vosdroits/F102, consultée le 13 juillet 2016.) Faute d'avoir atteint $10 \%$, le syndicat peut désigner un candidat qui a réalisé un score moindre, ou un de ses adhérents dans l'entreprise ou dans l'établissement. 
Les résultats statistiques témoignent de l'ambivalence du sentiment de discrimination déclaré dans le cadre de l'enquête statistique. En effet, la part des établissements où le représentant syndiqué interrogé indique que le mandat le protège du risque de perdre son emploi est du même ordre $(11 \%)$ que celle des établissements où il est perçu comme une menace (respectivement $12 \%$ et $13 \%$ pour les élus et désignés). Cette ambivalence reflète que leur sentiment d'être protégé ou exposé dans l'exercice de leur mandat ne se construit pas uniquement par rapport aux types de discrimination identifiés et sanctionnés par la loi. C'est également ce que tend à suggérer un second enseignement majeur de l'enquête REPONSE : c'est dans les grands établissements où les institutions représentatives du personnel sont les plus présentes et où les relations professionnelles sont les plus encadrées juridiquement que le sentiment de discrimination est le plus fréquent.

\section{Un sentiment de discrimination plus souvent déclaré dans les grands établissements}

On pourrait s'attendre à ce que ce soit dans les petits établissements que les représentants soient les plus nombreux à déclarer que leur engagement est un frein à leur carrière professionnelle ou un risque pour leur emploi. De fait, c'est dans les petits établissements que les obligations légales en matière de représentation et de consultation des représentants du personnel sont le moins souvent appliquées et que l'implantation syndicale est la plus fragile. Les pratiques de répression anti-syndicale (isolement professionnel, entrave au bon déroulement des carrières professionnelles, etc.) y sont d'ailleurs fréquemment utilisées par les directions afin d'empêcher la structuration du syndicalisme (DENIS, 2009). Dans les grands établissements, les instances de représentation sont en revanche plus solidement instituées et les relations entre représentants et dirigeants plus formalisées - du fait de la présence plus fréquente de syndicats fortement implantés d'une part, et de services de ressources humaines plus professionnalisés d'autre part. Les dirigeants des grands établissements sont en outre généralement plus enclins à tenir un discours valorisant le rôle des syndicats comme des «partenaires sociaux » avec lesquels il est nécessaire de « dialoguer » pour « fluidifier» les relations sociales (GIRAUD, 2007). C'est pourtant dans les grands établissements que les représentants de la liste majoritaire ont le plus souvent tendance à percevoir leur engagement comme un risque pour leur carrière professionnelle et leur emploi (voir tableau 3).

De manière combinée, la part des établissements où le représentant qui appartient à la liste syndicale majoritaire exprime un sentiment de discrimination est plus importante quand l'établissement appartient à un groupe (28\% quand le représentant est simplement un élu syndiqué et $39 \%$ lorsqu'il est délégué syndical) que quand il est franchisé ( $24 \%$ et $24 \%$ ) ou indépendant ( $20 \%$ et $29 \%$ ). Ce résultat se confirme toutes choses égales par ailleurs, l'effet de l'appartenance à un groupe étant particulièrement marqué statistiquement, au point qu'il semble même prévaloir sur la taille de l'établissement (voir tableau 4). Par ailleurs, c'est dans l'industrie, lieu d'implantation 
TABLEAU 3 - Sentiment que le mandat de représentant du personnel a été un frein à sa carrière selon la taille, le secteur et le type de propriétaire des établissements

$\%$ d'établissements

\begin{tabular}{lccc}
\hline & Élus non syndiqués & Élus syndiqués* $^{*}$ & Délégués syndicaux \\
\hline Taille (nombre de salariés) & 4 & 23 & 30 \\
$11-49$ & 4 & 22 & 32 \\
$50-99$ & 7 & 33 & 40 \\
$100-499$ & 12 & 32 & 47 \\
500 et plus & 1 & & \\
\hline Secteur & 5 & 19 & 16 \\
BTP & 4 & 32 & 33 \\
Commerce & 5 & 26 & 47 \\
Industrie & & & 30 \\
Services & 1 & 24 & 24 \\
Type de propriétaire & 8 & 28 & 39 \\
Franchise & 2 & 20 & 29 \\
Groupe & & & \\
Indépendant & & & \\
\hline
\end{tabular}

* Élus du personnel qui sont syndiqués mais n'exercent pas de fonction de délégué syndical.

Lecture : dans $32 \%$ des établissements de 500 salariés et plus où il y a des représentants du personnel élus et où la liste majoritaire est syndicale, le représentant du personnel interrogé déclare que son mandat est un frein pour sa carrière contre $22 \%$ des établissements de 11 à 49 salariés.

Champ : établissements d'au moins 11 salariés du secteur marchand non agricole.

Source : REPONSE, 2011, volets « employeurs » et « représentants du personnel » appariés, Dares ( $\mathrm{n}=2433)$.

historique du syndicalisme que le sentiment de discrimination est le plus fréquemment exprimé, notamment en ce qui concerne le déroulement de la carrière professionnelle. Cependant, une fois pris en compte la taille et le type de propriétaire de l'établissement, les différences entre secteurs ne sont plus significatives. Autrement dit, l'intensité du sentiment de discrimination est d'abord liée à la morphologie des entreprises et à leur structure capitalistique.

La corrélation avec la taille s'explique de plusieurs manières. On peut d'abord faire l'hypothèse que la perception des discriminations syndicales est plus difficile à repérer dans les petits établissements au moyen de l'enquête REPONSE. De fait, on le sait, la part des établissements de moins de 50 salariés dotés d'institutions représentatives est faible. Et même si la crainte des représailles patronales n'est pas le seul facteur explicatif de cette situation, on imagine néanmoins que la crainte d'être discriminé n'est pas absente dans cette catégorie d'établissements et qu'elle dissuade plus souvent que dans les grandes unités de s'engager dans un mandat de représentant du personnel. Par ailleurs, dans les grands établissements, la formation d'un sentiment de discrimination est logiquement favorisée dès lors que les possibilités de promotion professionnelle auxquelles les représentants du personnel sont susceptibles d'aspirer sont plus importantes que dans les petits établissements. En outre, le sentiment d'être désavantagé du fait de ses activités militantes dépend aussi de la comparaison que les 
TABLEAU 4 - Se sentir menacé dans son emploi ou freiné dans sa carrière selon les caractéristiques du représentant du personnel et celles de son établissement (régression logistique)

Rapport de chance

\begin{tabular}{|c|c|c|}
\hline & $\begin{array}{l}\text { Menace dans } \\
\text { son emploi }\end{array}$ & $\begin{array}{l}\text { Frein dans } \\
\text { sa carrière }\end{array}$ \\
\hline \multicolumn{3}{|l|}{ Sexe } \\
\hline Homme & réf. & réf. \\
\hline Femme & 1,1 & 0,8 \\
\hline \multicolumn{3}{|l|}{ Âge } \\
\hline Moins de 35 ans & 0,6 & $0,5 * * *$ \\
\hline 35-50 ans & réf. & réf. \\
\hline Plus de 50 ans & 0,8 & $0,8^{*}$ \\
\hline \multicolumn{3}{|l|}{ Diplôme } \\
\hline Moins que le bac & 0,9 & $0,7 *$ \\
\hline$B a c$ & réf. & réf. \\
\hline Plus que le bac & 1,3 & 0,9 \\
\hline \multicolumn{3}{|l|}{ Position professionnelle } \\
\hline Cadre & 1,1 & 0,9 \\
\hline Employé & 1,4 & 1,1 \\
\hline Technicien & réf. & réf. \\
\hline Ouvrier qualifié & 1,6 & $1,4^{*}$ \\
\hline Ouvrier spécialisé & 1,2 & 1,2 \\
\hline Qualification inconnue & 1,5 & 1,5 \\
\hline \multicolumn{3}{|l|}{ Mandat exercé } \\
\hline Non syndiqué & réf. & réf. \\
\hline Syndiqué & $6,6^{* * *}$ & $5,1 * * *$ \\
\hline Délégué syndical & $6,3 * * *$ & $7,6 * * *$ \\
\hline \multicolumn{3}{|c|}{ Institutions représentatives de l'établissement } \\
\hline Moins de deux & réf. & réf. \\
\hline Au moins deux & 1,3 & 1,1 \\
\hline \multicolumn{3}{|l|}{ Taille } \\
\hline Moins de 50 salariés & 1,5 & 0,9 \\
\hline 50-99 salariés & réf. & réf. \\
\hline 100-499 salariés & 1,4 & $1,5^{*}$ \\
\hline 500 salariés et + & 1,2 & $1,8 * * *$ \\
\hline \multicolumn{3}{|l|}{ Secteur } \\
\hline ВTP & 1,4 & 0,8 \\
\hline Commerce & 1 & 0,9 \\
\hline Services & 1 & 0,8 \\
\hline Industrie & réf. & réf. \\
\hline \multicolumn{3}{|l|}{ Type de propriétaire } \\
\hline Indépendant & réf. & réf. \\
\hline Franchise & 0,9 & 0,8 \\
\hline Groupe & $1,3^{*}$ & $1,6 * * *$ \\
\hline$\%$ de paires concordantes & 69,6 & 76,2 \\
\hline
\end{tabular}

* significativement différent de 0 ou de 1 au seuil $10 \%$,** significativement différent de 0 ou de 1 au seuil $5 \%$,*** significativement différent de 0 ou de 1 au seuil $1 \%$.

Méthode : les modélisations ont été effectuées avec une pondération normalisée et sans pondération. Dans les deux cas, le sens des coefficients est identique et la significativité des variables similaire. Nous avons gardé les modélisations sans pondération.

Lecture : à variables contrôlées, un délégué syndical a une probabilité 6,3 fois plus élevée qu'un élu non syndiqué de se déclarer menacé dans son emploi plutôt que protégé ou ni l'un ni l'autre.

Champ : représentant du personnel majoritaire des établissements du secteur marchand non agricole.

Source : REPONSE, 2011, volets « employeurs » et « représentants du personnel » appariés, Dares $(\mathrm{n}=2414)$. Ont été supprimés du champ les établissements pour lesquels trop d'informations étaient non renseignées $(n=19)$. 
représentants peuvent établir entre leur situation professionnelle et salariale et celle d'autres collègues non syndiqués. Or, la probabilité que les représentants se perçoivent désavantagés par rapport à leurs collègues est d'autant plus forte que les critères de gestion du personnel sont formalisés : c'est notamment le cas lorsque les carrières et les niveaux de qualification sont très codifiés, comme dans l'industrie. V.-A. CHAPPE (2013) indique d'ailleurs que c'est dans de grandes entreprises industrielles que les premières luttes contre la discrimination salariale des syndicalistes ont été engagées, justement parce qu'il était possible de comparer les carrières de cohortes de syndicalistes et de non syndicalistes au regard des grilles de qualification.

Surtout, on peut logiquement établir un lien entre ce sentiment de discrimination des délégués syndicaux dans les grands établissements et la plus forte intensité de l'action de représentation du personnel qui y est observée. En effet, pour qu'il y ait sentiment de discrimination syndicale, encore faut-il qu'il existe une réelle activité syndicale dans l'établissement et que les représentants expérimentent des situations de confrontation avec les directions d'entreprise. Or, c'est dans les grands établissements que l'on trouve les représentants les plus investis dans leurs mandats, que ce soit du point de vue des actions qu'ils mènent auprès des salariés ou des ressources qu'ils mobilisent (formation, appel à des experts) (PAK, PIGNONI, 2014). Ils prennent également part à un plus grand nombre d'échanges institutionnels avec la direction, du fait des nombreuses obligations de négociation et de consultation qu'impose la loi pour les entreprises de plus de 50 salariés.

On sait en outre que dans ces établissements, avec l'accroissement des dispositifs de négociation, la conflictualité collective demeure beaucoup plus fréquente que dans les plus petits établissements (Giraud et al., 2014 ; PignONI, RAYNAUd, 2013). On peut alors faire l'hypothèse que le sentiment de discrimination s'exprime d'autant plus que les relations professionnelles sont conflictuelles. C'est ce que laissent penser les données de l'enquête statistique, puisque dans les établissements ayant connu une grève lors des deux dernières années, ce sentiment est plus courant - surtout dans ceux où le représentant interrogé est délégué syndical - de même que dans ceux où l'activité de négociation a été intense (voir tableau $5^{7}$ ). Dans les établissements où le climat social est perçu comme tendu par les représentants du personnel, leur propension à se sentir discriminés est également plus élevée (32\% pour les élus syndiqués, $44 \%$ pour les délégués syndicaux ; contre respectivement $16 \%$ et $21 \%$ quand le climat social est jugé calme). Ces constats sont à mettre en perspective avec ce qu'établissent J. BouRDIEU et T. BREDA dans ce numéro, en montrant que les pénalités salariales subies par les représentants du personnel sont plus élevées dans les établissements ayant connu des grèves et des négociations collectives. De la même manière que le développement du « dialogue social » n'implique pas une déconflictualisation des relations professionnelles (BÉROUD et al., 2008), il ne protège pas nécessairement les élus syndiqués contre des formes objectives de discrimination salariale.

7. Voir également le tableau 6 qui se rapporte à la menace sur la stabilité de l'emploi. 
TABLEAU 5 - Sentiment que le mandat de représentant du personnel a été un frein à sa carrière selon l'état des relations professionnelles dans l'établissement

$\%$ d'établissements

\begin{tabular}{lccc}
\hline & Élus non syndiqués & Élus syndiqués* & Délégués syndicaux \\
\hline Conflits (selon le dirigeant) & 9 & & \\
Grève entre 2008 et 2010 & 4 & 26 & 43 \\
Absence de grève entre 2008 et 2010 & 24 & 31 \\
\hline
\end{tabular}

Négociation sur les salaires (selon le dirigeant)

\begin{tabular}{llll} 
Négociation en 2010 & 6 & 26 & 39 \\
Pas de négociation en 2010 & 3 & 22 & 25 \\
\hline
\end{tabular}

Intensité de négociations ou discussions

d'entreprise autres que salariales ${ }^{* *}$

(selon le dirigeant)

Forte (plus de 7 thèmes de négociations)

Moyenne (4 à 7 thèmes de négociations)

$4 \quad 36 \quad 41$

Faible (moins de 4 thèmes de négociations)

$8 \quad 20 \quad 36$

Opinion du dirigeant sur les syndicats

(« les syndicats gênent le déroulement

des activités de l'entreprise »)

Tout à fait / plutôt d'accord

$3 \quad 17$

27

Pas du tout / plutôt pas d'accord

$3 \quad 24 \quad 43$

Opinion du dirigeant sur les

représentants ( les RP traduisent bien

les aspirations et revendications

des salariés »)

Tout à fait / plutôt d'accord

$4 \quad 20 \quad 28$

Pas du tout / plutôt pas d'accord

$7 \quad 41$

56

Climat social (selon le représentant)

$\begin{array}{lrrr}\text { Calme } & 2 & 16 & 21 \\ \text { Tendu } & 11 & 32 & 44\end{array}$

Entrave au droit syndical

(selon le représentant)

Procédure juridique

12

38

58

Pas de procédure

4

23

29

* Élus du personnel qui sont syndiqués mais n'exercent pas de fonction de délégué syndical.

** Les thèmes de discussions ou négociations possibles sont au nombre de dix : le temps de travail, les qualifications ou classifications, l'emploi, les conditions de travail, les changements technologiques ou organisationnels, la formation professionnelle, le droit d'expression des salariés ou droit syndical, l'égalité professionnelle, l'épargne salariale, la protection sociale complémentaire.

Lecture : dans $43 \%$ des établissements ayant connu une grève (selon le dirigeant) où il y a des représentants du personnel désignés par une organisation syndicale, le représentant du personnel interrogé (désigné par l'organisation comptant le plus de délégués syndicaux) déclare que son mandat est un frein pour sa carrière, contre $31 \%$ des établissements n'ayant pas connu de grève.

Champ : établissements d'au moins 11 salariés du secteur marchand non agricole.

Source : REPONSE, 2011, volets « employeurs » et « représentants du personnel » appariés, Dares $(\mathrm{n}=2433)$.

Les effets du contexte conflictuel des relations sociales dans la construction du sentiment de discrimination peuvent être mieux compris lorsque l'on considère la perception que représentants du personnel et dirigeants d'établissement se font de leur interlocuteur dans le jeu des relations professionnelles. C'est dans les établissements où 
TABLEAU 6 - Sentiment que le mandat de représentant du personnel a été une menace pour son emploi selon l'état des relations professionnelles dans l'établissement

$\%$ d'établissements

\begin{tabular}{lccc}
\hline & Élus non syndiqués & Élus syndiqués & Délégués syndicaux \\
\hline Conflits (selon le dirigeant) & & & \\
Grève entre 2008 et 2010 & 0 & 17 & 14 \\
Absence de grève entre 2008 et 2010 & 2 & 10 & 12 \\
\hline
\end{tabular}

Négociation sur les salaires

(selon le dirigeant)

\begin{tabular}{llll} 
Négociation en 2010 & 3 & 13 & 13 \\
Pas de négociation en 2010 & 1 & 11 & 13 \\
\hline
\end{tabular}

Intensité de négociations ou discussions

d'entreprise autres que salariales

(selon le dirigeant)

Forte (plus de 7 thèmes de négociations)

\begin{tabular}{rrr}
3 & 15 & 13 \\
5 & 12 & 17 \\
1 & 9 & 8 \\
\hline
\end{tabular}

Moyenne (4 à 7 thèmes de négociations)

Faible (moins de 4 thèmes de négociations)

Opinion du dirigeant sur les syndicats

(« les syndicats gênent le déroulement

des activités de l'entreprise »)

Tout à fait / plutôt d'accord

$\begin{array}{lll}1 & 16 & 16\end{array}$

Pas du tout / plutôt pas d'accord

211

12

Opinion du dirigeant sur les

représentants ( « les RP traduisent bien

les aspirations et revendications

des salariés »)

Tout à fait / plutôt d'accord

$\begin{array}{lll}2 & 10 & 11\end{array}$

Pas du tout / plutôt pas d'accord

219

19

Climat social (selon le représentant)

$\begin{array}{lrrr}\text { Calme } & 0 & 8 & 5 \\ \text { Tendu } & 6 & 16 & 18\end{array}$

Entrave au droit syndical

(selon le représentant)

Procédure juridique

$\begin{array}{lll}5 & 22 & 24 \\ 2 & 11 & 11\end{array}$

Pas de procédure

5

* Élus du personnel qui sont syndiqués mais n'exercent pas de fonction de délégué syndical.

Lecture : dans $16 \%$ des établissements avec un climat social tendu où il y a des représentants du personnel élus et où la liste majoritaire est syndicale, le représentant du personnel déclare que son mandat est une menace pour son emploi, contre $8 \%$ des établissements avec un climat social calme.

Champ : établissements d'au moins 11 salariés du secteur marchand non agricole.

Source : REPONSE, 2011, volets « employeurs » et « représentants du personnel » appariés, Dares $(\mathrm{n}=2433)$.

les prises de position des dirigeants sont les moins bienveillantes envers les syndicats et les représentants du personnel que les élus et délégués syndicaux ont davantage tendance à se déclarer discriminés. C'est notamment le cas quand le dirigeant interrogé estime que les syndicats sont gênants pour l'entreprise ou que les représentants du 
personnel ne traduisent pas les aspirations des salariés (voir tableau 6). Là encore, ces résultats font écho à l'étude de V.-A. CHAPPE (2013) qui montre que les luttes contre la discrimination syndicale se sont d'abord développées dans le secteur automobile, notamment chez Peugeot, marqué historiquement par des confrontations importantes entre les délégués syndicaux de la CGT et la direction de l'entreprise ainsi que par des conflits collectifs importants (l'article de Nicolas HATZFELD dans ce numéro en retrace l'histoire).

On retrouve ce cas de figure dans deux monographies que nous avons réalisées, Transformateur et Engins, qui sont deux grands établissements industriels. Dans le second, M. Cluzel, délégué syndical de la CGT (majoritaire), a déclaré que son mandat avait nui à sa carrière professionnelle. Recruté comme ouvrier au milieu des années 2000, il a pourtant obtenu en 2012 un poste hors de la chaîne de production, comme « animateur qualité ». Sa perception de la discrimination renvoie à l'évaluation qu'il fait de l'ensemble de sa carrière professionnelle autant qu'à sa position actuelle chez Engins. Âgé de 45 ans, il a occupé plusieurs postes d'ouvrier dans des grands groupes de l'industrie ou du bâtiment et des travaux publics (BTP $)^{8}$ et s'est engagé dès ses 20 ans à la CGT; il a ainsi exercé en tant que délégué syndical des entreprises dans lesquelles il a travaillé mais aussi en tant que secrétaire d'union locale. Très actif, même s'il prône un syndicalisme réformiste et de négociation - critiquant la posture des délégués syndicaux cégétistes trop « anti-patrons »-, il estime que cet engagement lui a valu de nombreuses difficultés tant professionnelles que familiales. Il insiste particulièrement sur le fait qu'il a refusé plusieurs fois des « propositions de promotion », qu'il considère comme autant de tentatives pour l'acheter, et met en avant le fait que son professionnalisme - «j' ai toujours eu le cul propre » - ait été reconnu par ses supérieurs. En entretien, tandis que ce militant relate longuement les nombreux points de conflits avec la direction qui émaillent le fonctionnement routinier des institutions représentatives et qui ont donné lieu à plusieurs débrayages ces dernières années, un autre de ses collègues, M. Brénot, ancien secrétaire de la section CGT recruté en 1967 comme apprenti sur un poste d'ajusteur et ayant fait toute sa carrière dans l'usine comme ouvrier, revient plus spécifiquement sur ce qu'il décrit comme une politique de discrimination ciblant les syndicalistes de la CGT. Recruté parce que son père, cégétiste, travaillait déjà dans l'usine, il est présent au milieu des années 1980, lorsqu'une multinationale américaine rachète l'usine. Alors syndiqué à la CGT, il se rappelle qu'il a vu la grande majorité de ses collègues du syndicat être licenciés dans le cadre du plan social ayant fait suite à ce rachat. C'est d'ailleurs ce qui explique, selon lui, que dans les années 1990, la Confédération française démocratique du travail (CFDT) est restée majoritaire dans l'usine au détriment de la CGT. Se considérant lui-même victime de discrimination en raison de son engagement syndical - «j'ai formé plusieurs jeunes qui me sont passés bien devant côté salaire »-, il explique 
avoir constitué un dossier pour faire constater juridiquement cette situation, finalement reconnue officieusement par la direction :

M. Brénot vient d'expliquer que sous l'ancien propriétaire de l'usine, il n'avait pas connu de faits de discrimination syndicale. Le rachat de l'usine par un grand groupe américain change les rapports avec la direction.

«Alors quand Engins a racheté l'usine, vous avez l'impression qu'il y a eu une politique plus dure vis-à-vis des syndicats ?»

M. Brénot : «Eh bien, disons qu'au niveau des syndiqués et des représentants du personnel, on n'avait pas le droit à grand-chose au niveau des augmentations. »

«Avec A. (ancien patron) vous étiez augmenté comme les autres alors qu'au niveau de Engins...»

« Oui c'est ça. J'ai monté tout un dossier mais comme j'ai fait un accord avec la direction, je ne peux pas trop le dire... Il a fallu que je me batte pour leur démontrer que pendant les vingt premières années, $\mathrm{j}$ 'avais toujours eu de l'augmentation, toujours eu un changement de coeff[icient], etc. et puis arrivé en 86 je n'ai plus jamais rien eu. »

«C'est plus ce que l'on peut appeler de la discrimination syndicale?»

«Voilà. »

«Et ça a duré jusqu'à récemment? »

« Oui, on a réussi en se battant parce qu'on a fait des grèves assez dures pendant deux, trois jours, alors qu'on n'avait jamais fait grève, rien du tout depuis un moment, et pas pour de l'augmentation mais pour du respect...»

« [...] Et vous aviez monté un dossier ?»

« Oui j'avais monté un dossier avec mes collègues qui sont rentrés en même temps que moi. Certains autres syndiqués d'autres syndicats ont fini leur carrière avec un grade deux ou trois fois supérieur au mien. »

(Entretien réalisé le 4 février 2013)

La lutte contre les discriminations syndicales a ainsi marqué l'histoire récente de cet établissement, comme de beaucoup d'autres grands établissements industriels (Peugeot, Renault, Airbus, Dassault, EADS, SNPE, Tracma, etc.). L'expression plus fréquente d'un sentiment de discrimination dans ces grands établissements pourrait signifier le maintien de pratiques de discrimination et de répression syndicales malgré le développement du dialogue social (WiLlEMEZ, 2012). De fait, chez Engins comme chez Transformateur, la lutte contre les discriminations à l'encontre des syndicalistes semble rester un motif de conflit important entre les représentants syndicaux et les directions de ces établissements. M. Henri (délégué CGT chez Transformateur) explique par exemple qu'il lui faut régulièrement réclamer les primes auxquelles il estime avoir droit : «Il faut toujours que je sois vigilant, sinon, dès qu'ils peuvent me carotter, ils me carottent » (entretien réalisé le 22 mai 2013). 
Toutefois, il reste difficile, au regard des seules données de l'enquête REPONSE, d'établir fermement le lien entre discrimination objective et sentiment de discrimination, encore plus lorsqu'on élargit la question aux entraves à l'action syndicale. Avec l'appui des enquêtes de terrain, il est en revanche possible d'explorer d'autres pistes d'interprétation des résultats de l'enquête statistique en dépassant certains de ses angles morts. Les monographies d'entreprise permettent en particulier de mieux appréhender la diversité des raisons pour lesquelles des représentants peuvent se déclarer discriminés, et de questionner les conditions qui facilitent l'usage de la catégorie de discrimination dans les grands établissements.

\section{Le caractère pluridimensionnel et relationnel du sentiment de discrimination}

Le recours à des enquêtes monographiques permet en premier lieu d'aller au-delà d'une double limite à laquelle se heurte l'enquête statistique dans sa manière d'appréhender le phénomène des discriminations syndicales. Celle-ci ne s'intéresse en effet qu'aux formes individuelles de discrimination, mesurées au moyen de questions centrées sur les effets perçus de l'exercice d'un mandat représentatif sur la carrière professionnelle. De plus, seules les déclarations d'un représentant de la liste majoritaire sont recueillies. Cette approche statistique a pour inconvénient d'occulter la variété des motifs pour lesquels les représentants peuvent se sentir discriminés. Elle tend aussi à masquer la diversité des points de vue que peuvent exprimer les représentants, en fonction de la position qu' ils occupent dans l'espace de la représentation du personnel de leur établissement et de leurs manières de s'engager dans leur mandat.

\section{Discrimination individuelle et entrave au droit syndical : des plaintes qui se combinent}

Des entretiens réalisés dans les établissements où nous avons enquêté, il ressort tout d'abord que, dans les discours des représentants qui se plaignent de discrimination, il n'est pas uniquement fait référence à des situations de discrimination individuelle, telles qu'elles sont sanctionnées par le droit. Ce qui est mis en cause de manière récurrente par ces représentants, c'est aussi - et parfois surtout - le fait de se sentir entravés dans l'exercice de leur mandat et de leurs droits syndicaux par le maintien de stratégies managériales de domestication de l'action syndicale. Tel est le cas, par exemple, dans l'établissement Engins, qui fabrique des machines utilisées dans les travaux publics. En entretien, M. Cluzel (délégué CGT) souligne surtout que ses camarades du syndicat et lui-même continuent d'être pénalisés dans l'exercice de leurs droits de représentation du personnel, accusant la direction d'avoir injustement contesté les résultats électoraux de leur organisation et de faire obstacle à la mise en œuvre des règles légales de fonctionnement des instances (fixation de l'ordre du jour des réunions, transmission 
des informations et des documents, etc.). Le cas de l'établissement Transformateur illustre également la manière dont se combinent et se renforcent des motifs de plainte de différents ordres dans le sentiment de discrimination qu'exprime le représentant du personnel. De fait, M. Henri (également délégué CGT) se plaint d'abord d'être soumis à une pression constante de sa hiérarchie, qui le contraint à devoir défendre ses droits :

« Pour moi, c'était clairement défini qu'il y avait discrimination, puisque même dans les entretiens, on m'en a parlé. Mon chef m’a dit : "Le syndicat, je t'avais prévenu, tu vas pas évoluer énormément." Je lui ai dit: "Bah, je ne vois pas pourquoi je ne pourrais pas évoluer énormément.” Si c'est une entreprise honnête, ils vont me faire évoluer par rapport à mon travail, le syndicat n'a rien à voir ; je veux dire M. Henri monteur, c'est différent de M. Henri mandaté par le syndicat. Mais eux, ils font l'amalgame des deux, c'est la même personne pour eux. J'ai dû aller chercher deux fois des rallonges et une fois un coeff[icient]. J'ai eu affaire à des chefs au-dessus des responsables de prod[uction], etc., et on m'a dit clairement dans les entretiens que le mandat syndical, les heures de délégation que je prenais, ça leur posait problème. »

(Entretien réalisé le 22 mai 2013)

Le sentiment de M. Henri d'être exposé à des risques de représailles patronales est redoublé par les relations conflictuelles qu'il entretient avec sa direction dans les instances de représentation et lors des négociations collectives. Les syndicalistes de l'établissement, ceux du syndicat majoritaire (CGT) comme ceux des organisations concurrentes (CFDT, UNSA [Union nationale des syndicats autonomes]), sont en effet unanimes pour dénoncer un « dialogue social » de façade, accusant la direction de se contenter de se conformer à ses obligations légales de réunir les représentants, sans pour autant être disposée à entrer dans une véritable logique d'échanges et de discussions : «La négo[ciation], ici, c'est : je décide, vous m'écoutez ! (M. Henri). Dans ce contexte de relations tendues, ce dernier accuse les dirigeants de chercher à l'empêcher de tenir son rôle de représentant du personnel. Il leur reproche, par exemple, d'avoir voulu lui interdire de faire appel à un expert dans le cadre du comité d'hygiène, de sécurité et des conditions de travail (CHSCT), de s'opposer à son travail de défense des salariés convoqués pour des entretiens disciplinaires, ou bien encore de faire circuler dans l'usine des rumeurs malveillantes à son encontre.

Le sentiment de discrimination exprimé par ces représentants n'implique pas qu'ils subissent objectivement des situations de discrimination individuelle, telles qu'elles sont sanctionnées par la loi, ne serait-ce que parce que les représentants parviennent dans une certaine mesure, par leur mobilisation, à empêcher la persistance de telles pratiques patronales. La discrimination ici évoquée renvoie largement au sentiment que la reconnaissance de leurs droits individuels par la direction reste un enjeu de lutte et, plus globalement, aux dimensions plus collectives de leur action de représentants. C'est la persistance de ce qui est perçu comme des pratiques d'entrave aux droits syndicaux que ces militants ressentent comme pénalisante et répressive. L'enquête REPONSE ne comporte aucune question portant spécifiquement sur ces stratégies managériales. Elles sont, de fait, difficiles à saisir par questionnaire, tant 
elles sont aux frontières de la légalité. Néanmoins, la probabilité que le représentant interrogé déclare se sentir discriminé est plus élevée, toutes choses égales par ailleurs, dans les établissements où il a par ailleurs indiqué avoir initié une procédure juridique pour entrave aux droits syndicaux (voir tableau 7).

\section{TABLEAU 7 - Se sentir menacé dans son emploi ou freiné dans sa carrière selon l'état des relations professionnelles et les pratiques du représentant du personnel (régression logistique)}

Rapport de chance

\begin{tabular}{ccc}
\hline & Menace dans & Frein dans \\
sa carrière
\end{tabular}

Conflit (selon le dirigeant)

Grève entre 2008 et 2010

$0,9 \quad 1,2$

Absence de grève entre 2008 et 2010

réf.

réf.

Négociation sur les salaires (selon le dirigeant)

Négociation en 2010

Pas de négociation en 2010

$11,4 * *$

Entrave au droit syndical (selon le représentant)

réf.

réf.

Procédure juridique

Pas de procédure

$1,9 * * *$

réf.

\section{Climat social (selon le représentant)}

Tendu

Calme

\section{$3,1 * * *$}

réf.

\section{$1,6 * * *$}

réf.

Temps passé par le représentant du personnel dans l'animation des conflits (au cours des 3 dernières années)

Beaucoup ou presque tout son temps

Peu ou pas de temps

$1,8 * * *$

réf.

0,5
$2,2 * * *$

réf.

Temps non connu

Temps passé par le représentant du personnel dans la négociation d'accords collectifs (au cours des 3 dernières années)

Beaucoup ou presque tout son temps

$0,9 \quad 0,8^{*}$

Peu ou pas de temps

réf.

réf.

Temps non connu

1,4

$\%$ de paires concordantes

77,9

1,3

* significativement différent de 0 ou de 1 au seuil $10 \%$,** significativement différent de 0 ou de 1 au seuil $5 \%$,*** significativement différent de 0 ou de 1 au seuil $1 \%$.

Méthode : les modélisations ont été effectuées avec une pondération normalisée et sans pondération. Dans les deux cas, le sens des coefficients est identique et la significativité des variables similaire. Nous avons gardé les modélisations sans pondération.

Lecture : à variables contrôlées, un représentant du personnel ayant déclaré passer beaucoup ou tout son temps dans l'animation de conflits a une probabilité 1,8 fois plus élevée qu'un représentant du personnel ayant déclaré passer peu ou pas de temps dans l'animation de conflits de se déclarer menacé dans son emploi. Outre celles présentées ici, les variables de contrôle sont celles du tableau 4 (sexe, qualification, âge, diplôme et syndicalisation du représentant ainsi que la taille, le secteur, le nombre d'institutions représentatives du personnel et le type de propriétaire de l'établissement).

Champ : représentant du personnel majoritaire des établissements du secteur marchand non agricole.

Source : REPONSE, 2011, volets « employeurs » et « représentants du personnel » appariés, Dares $(\mathrm{n}=2414)$. Ont été supprimés du champ les établissements pour lesquels trop d'informations étaient non renseignées $(\mathrm{n}=19)$. 
La mise en relation, dans le discours des représentants, de la discrimination individuelle et des entraves à l'exercice des droits collectifs - pensées comme un continuum de stratégies patronales de domestication de l'action syndicale - s'opère d'autant plus facilement dans le contexte des grands établissements que les institutions représentatives y sont plus souvent investies par des militants syndicaux activement engagés dans leur mandat et aguerris à l'usage du droit. La plus forte propension de ces représentants à se déclarer discriminés peut alors se comprendre comme la traduction de la plus grande intensité des « conflits de règles » qui accompagnent le processus d'institutionnalisation des relations entre direction et représentants dans ces établissements (Groux, 1998).

\section{Un sentiment de discrimination dépendant des modes d'engagement des représentants dans leur mandat}

Les déclarations des représentants interrogés dans REPONSE - qui sont issus de l'organisation ou de la liste majoritaire - ne sont pas nécessairement représentatives du sentiment des autres représentants des établissements, notamment de ceux élus sur des listes minoritaires. De fait, les grands établissements ont plus de chances de connaître le pluralisme syndical. C'est une caractéristique importante du fonctionnement des relations sociales de ces établissements à prendre en compte si l'on souhaite mettre en évidence l'effet de pratiques militantes différentes et de positions concurrentes des syndicalistes sur leur inclination à se sentir (ou non) discriminés.

De fait, comme le suggère le cas de Transformateur, même dans les entreprises où l'ensemble des représentants syndiqués juge le climat social tendu, tous ne se sentent pas pour autant discriminés par leur direction. La mise en perspective du discours de M. Henri, délégué CGT dans cette usine, avec celui du secrétaire du comité d'entreprise (CE), affilié à la CFDT, est de ce point de vue éloquente. Alors que, comme nous l'avons vu précédemment, $\mathrm{M}$. Henri se plaint d'être exposé à une pression constante de la part de sa hiérarchie, le secrétaire du CE, M. Aziza, ne partage pas cet avis. Même s'il s'accorde lui aussi à penser que la direction n'est pas ouverte au « dialogue social », il estime pour autant ne pas rencontrer de difficultés particulières du fait de sa fonction :

« Dans cette entreprise, le rôle de secrétaire de CE, c'est assez facile pour l'instant, parce qu'on est dans une entreprise qui tourne bien. Non, c'est vraiment tranquille. »

(Entretien réalisé le 26 mai 2013)

Ces divergences de points de vue trouvent un début d'explication lorsqu'on les ramène aux pratiques syndicales différenciées des deux enquêtés. M. Henri est très impliqué dans son rôle de porte-parole des salariés : il est de toutes les négociations et apporte régulièrement son soutien aux collègues qui font appel à lui quand ils sont en conflit avec la hiérarchie. À l'occasion des négociations salariales de 2011, il fut l'un des principaux instigateurs de la première grève connue par l'établissement depuis plus de dix ans. Ce sont ses activités militantes et revendicatives qui, selon lui, l'exposent 
aux représailles de la direction. Il estime par exemple que son engagement dans la grève lui a valu de perdre le bénéfice d'une augmentation :

«J'avais une augmentation qui était censée tomber et puis elle n'est pas tombée.

Après, on m'a expliqué qu'elle n'était pas tombée parce qu'en haut lieu, ils avaient décidé de ne pas me la donner par rapport à la grève. »

(Entretien réalisé le 22 mai 2013)

Pour sa part, M. Aziza, consacre l'essentiel de son activité à la gestion des œuvres sociales. Il admet que de ce fait ses échanges avec la direction restent très ponctuels et consensuels, contrairement aux délégués syndicaux impliqués dans des relations plus fréquentes et conflictuelles avec la direction des ressources humaines (DRH). Au-delà du cas de cet établissement, les données de l'enquête REPONSE suggèrent que certaines pratiques militantes favorisent plus que d'autres l'émergence d'un sentiment de discrimination. Ce dernier est davantage exprimé par les représentants qui déclarent s'investir beaucoup dans l'animation des conflits (40\% pour les élus syndicaux de ces établissements, contre $21 \%$ lorsqu'ils s'investissent moins). En revanche, le sentiment de discrimination est tendanciellement moins élevé dans les établissements où le représentant interrogé déclare s'investir beaucoup dans les négociations (voir tableaux 8 et 9 ).

TABLEAU 8 - Sentiment que le mandat de représentant du personnel a été un frein à sa carrière selon les pratiques militantes déclarées et l'étiquette syndicale

$\%$ d'établissements

Élus non syndiqués Élus syndiqués* Délégués syndicaux

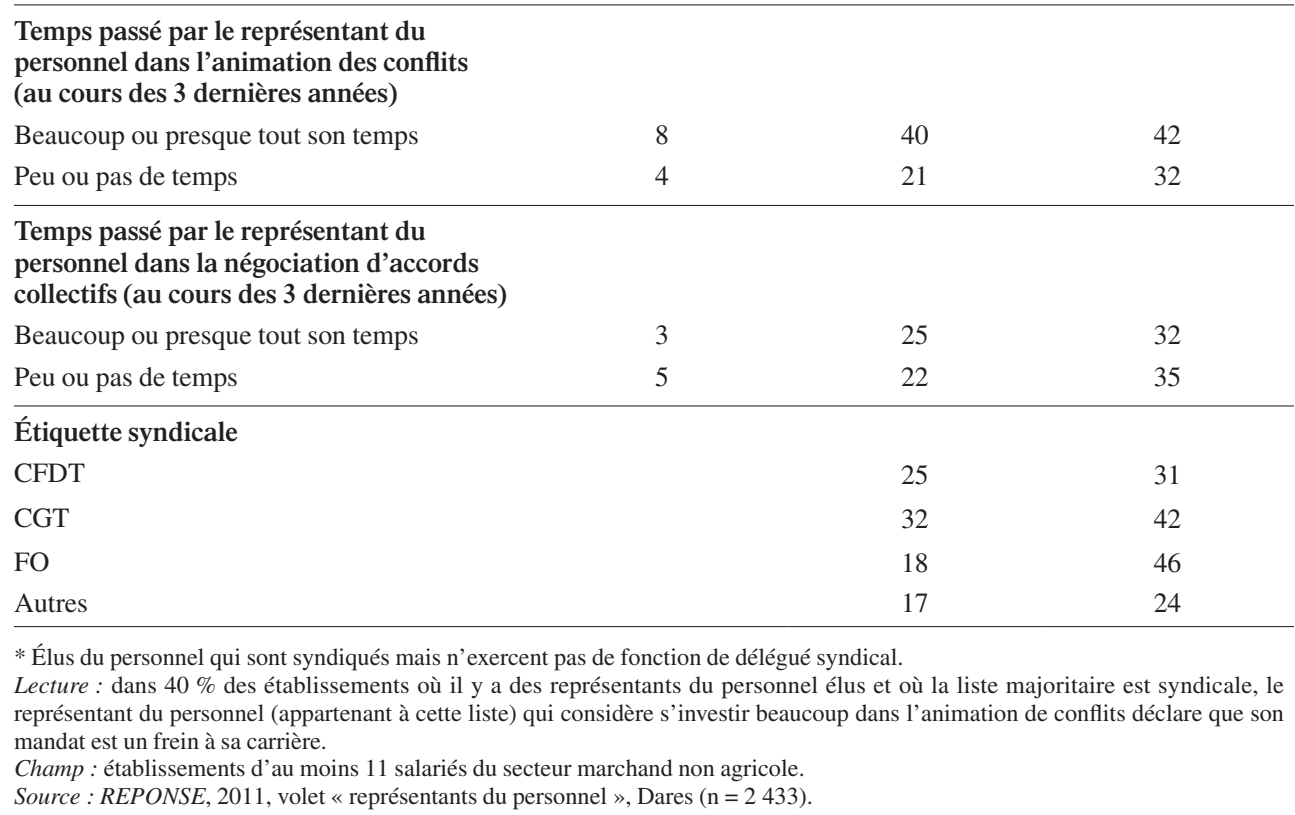


TABLEAU 9 - Sentiment que le mandat de représentant du personnel a été une menace pour son emploi selon les pratiques militantes déclarées et l'étiquette syndicale

$\%$ d'établissements

Élus non syndiqués Élus syndiqués* Délégués syndicaux

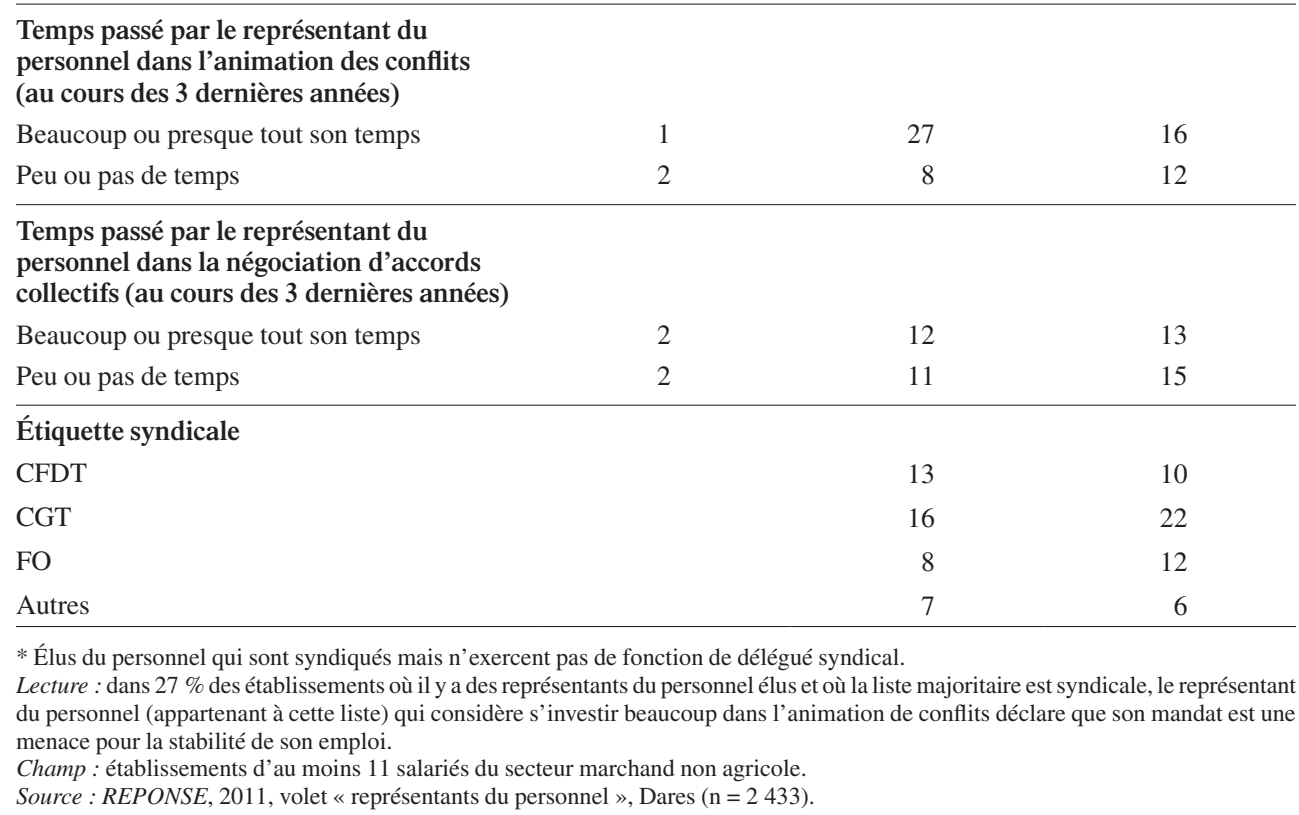

À l'image de ces deux militants syndicaux de Transformateur, la manière différente des représentants d'endosser leur rôle et de vivre leur engagement conduit à s'interroger plus largement quant aux effets de leur socialisation militante concurrente sur leur inégale propension à se déclarer discriminés. De fait, $M$. Henri semble incarner l'archétype du militant cégétiste acquis aux principes d'un "syndicalisme de lutte », axés sur la critique des antagonismes de classe (GANTOIs, 2014), et valorisant plus que d'autres l'idée selon laquelle la répression patronale participe de la logique des affrontements qui structurent le fonctionnement ordinaire des relations professionnelles. Il ressort d'ailleurs de l'enquête REPONSE que dans les établissements où le représentant majoritaire interrogé est affilié à la CGT, le sentiment de discrimination est plus fréquent (32\% pour les élus, $46 \%$ pour les désignés) que dans les établissements où il l'est à la CFDT (respectivement $25 \%$ et $31 \%$ ) (voir tableau 8). Pour autant, comme bien d' autres études l'ont montré (BÉROud et al., 2008 ; PIOTET, 2009), la CGT est - comme toutes les organisations - l'objet d'investissements militants très hétérogènes, et les adhérents s'affranchissent parfois largement des principes d'action revendiqués par l'organisation à laquelle ils sont affiliés. La diversité des modes d'engagement à la CGT est d'ailleurs très perceptible chez Transformateur. Tout en regrettant qu'une partie des adhérents de sa section soient inégalement impliqués 
dans leur mandat, M. Henri admet qu'ils ne s'exposent guère de ce fait au risque d'être sanctionnés par la direction de l'établissement :

«C'est un problème général (la discrimination) ? »

«Non, c'est quelque chose de nuancé. [...] Dans le syndicat, on a des mecs qui ne se font pas voir, qui ne vont pas au devant. Eux, par contre, ils ne sont pas emmerdés par la direction, mais ils prennent des heures, le personnel les voit pas à la boîte, personne sait même s'ils ont un mandat, ces gens-là, ils n'emmerdent personne et ils ne sont pas emmerdés! Par contre le mandaté, qui lui fait son boulot, creuse, va voir les collègues et s'investit dans son mandat, lui, il est emmerdé. »

(Entretien réalisé le 22 mai 2013)

De fait, la manière différente dont M. Henri et M. Aziza s'engagent dans leur mandat et perçoivent les risques qui lui sont liés ne saurait s'expliquer uniquement par leur affiliation syndicale. Elle est davantage révélatrice de la variété des conditions possibles d'engagement dans un mandat syndical et/ou d'élu, et des usages qui peuvent être faits de la protection juridique qu'il offre à ses titulaires. Messieurs Henri et Aziza présentent en effet deux trajectoires d'engagement très dissemblables. Le premier a été confronté très jeune au syndicalisme. Ouvrier, 35 ans, issu d'une famille dont plusieurs membres étaient eux-mêmes déjà syndiqués à la CGT, il a fait le choix d'y adhérer pour se protéger, on l'a dit, contre le risque d'éventuelles représailles patronales, qu'il dit avoir subies dans ses précédents emplois. Pour le second, l'entrée dans le syndicalisme procède d'une logique très différente. En effet, alors qu'il s'était toujours tenu en retrait de l'action des syndicats, cet ancien ouvrier devenu agent de maîtrise a accepté de figurer sur les listes de la CFDT «pour rendre service » à l'un de ses collègues, mais aussi pour se protéger de sa hiérarchie suite à un différend professionnel avec ses supérieurs : «Comme j'étais un peu dans un placard, que je ne faisais plus rien... Je me suis dit, tant qu'à être mal vu, autant être mal vu pour quelque chose, et être au moins protégé. » Loin de se sentir mis en danger par son mandat de secrétaire du comité d'entreprise, cet élu y voit ainsi, au contraire, une opportunité pour sécuriser sa position professionnelle.

Le cas de l'entreprise Transformateur montre ainsi que la variété des modes d'investissement des représentants dans leur mandat, le caractère plus ou moins conflictuel des relations qu'ils impliquent avec les dirigeants, et leurs manières différentes de faire usage du statut de salarié protégé contribuent à expliquer qu'ils ne perçoivent pas les mêmes risques à exercer leur mandat. À l'image de ce militant de la CGT, M. Henri, qui considère que sa direction est bien plus indulgente à l'égard des représentants du personnel qui ne la «gênent » pas, on comprend que la discrimination ressentie par les représentants dépend aussi de la façon dont ils comparent leurs situations : les pratiques de répression patronale viseraient prioritairement les pratiques syndicales les plus « contestataires ». C'est en tout cas ce que laisse penser l'observation des stages de formation des DRH à la gestion des relations sociales (GIRAUD, 2013). Ce résultat invite également à questionner, par le biais d'enquêtes qualitatives, l'effet des habitus militants (formes de politisation, types de rapports à l'engagement et 
aux pratiques syndicales, positions dans l'espace militant) sur l'inégale perception des discriminations ${ }^{9}$. Leur position dans l'espace syndical de l'entreprise en est une première illustration.

\section{Le sentiment de discrimination se nourrit de la comparaison entre syndicalistes}

Le point de vue des élus minoritaires recueilli chez Engins et Bus laisse entrevoir d'autres manières de se sentir lésés par rapport à ses concurrents syndicaux dans la façon dont la direction gère ses relations avec les syndicats. En fonction du point de vue qui s'exprime, la position de représentant syndical majoritaire peut être effectivement perçue de manière contradictoire : ressentie, par certains de ceux qui l'occupent, comme une position qui expose au risque d'être discriminé, elle peut être considérée au contraire, par les élus minoritaires, comme une position qui protège et facilite les relations avec la direction. Le sentiment de discrimination ne se limite donc pas nécessairement aux syndicalistes occupant une position dominante dans l'établissement et ne se détermine pas uniquement en fonction du degré d'investissement militant dans le mandat. Il varie aussi en fonction de la position relative (et mouvante) des représentants dans le jeu des rapports de force qui les opposent.

L'évolution du paysage syndical chez Engins à la fin des années 2000 en est une illustration. Comme le raconte M. Moreira, un jeune syndicaliste de la CGT élu au comité d'entreprise de cet établissement, plusieurs militants de l'organisation ont menacé pendant plus d'un an leur direction d'un recours juridique pour se plaindre de n'avoir obtenu aucune augmentation de salaire depuis leur entrée dans l'usine. Cette mobilisation leur a permis d'obtenir chacun un changement de coefficient, qui est présenté comme un « rattrapage ». Selon ce militant cégétiste, la décision a suscité « la jalousie de certains collègues, qui vont même jusqu'à considérer qu'on [les] a "achetés" ». Et lorsqu'on l'interroge, M. Benarfa ${ }^{10}$, délégué syndical Force ouvrière (FO), minoritaire dans l'établissement, laisse également entendre que les cégétistes sont mieux traités individuellement que lui, évoquant le fait que ce n'est qu'à l'issue de l'intervention de l'inspection du travail qu'il a échappé à un licenciement après une altercation avec un chef.

Ce renversement de la discrimination perçue s'explique en partie par la transformation du champ syndical dans l'usine et du rôle que la CGT y jouait. Longtemps

\footnotetext{
9. Les résultats de l'enquête Trajectoires et origines (TEO) montrent d'ailleurs que si le sentiment de discrimination syndicale et politique - c'est-à-dire en fonction de l'appartenance ou des opinions politiques et syndicales - est peu élevé dans la population (entre 1 et $2 \%$ ), il est très fortement corrélé au degré d'engagement : plus on participe à des activités militantes et plus on a de chances, toutes choses égales par ailleurs, de déclarer des formes de discrimination ou de stigmatisation (Algava, BÈQue, 2006).

10. Titulaire d'un certificat d'aptitude professionnelle (CAP) en électrotechnique, il a exercé comme manutentionnaire à l'aéroport de Roissy, avant d'être recruté chez Engins au début des années 2000 comme ajusteur monteur, en intérim puis en contrat à durée indéterminée. Avant de créer la section FO à la fin de la décennie, au motif que la CGT ne défend pas les jeunes ouvriers contre la hiérarchie, il n'avait pas d'expérience syndicale. Au moment de l'enquête, il a quitté la ligne de production pour devenir « agent logistique ».
} 
en concurrence avec la CFDT, syndicat majoritaire jusqu'au milieu des années 2000, les cégétistes occupaient dans l'usine une posture combative là où les élus de la CFDT incarnaient un pôle plus conciliateur. Avec la disparition de la CFDT, suite à la promotion de son principal animateur - qui est passé du collège des ouvriers à celui des cadres -, une partie des syndiqués de la CGT fait le choix d'endosser une nouvelle posture plus conciliatrice, encouragée par l'arrivée en 2007 de M. Cluzel. Cette posture est renforcée lors de la crise qui touche l'usine à partir de 2009 : face à la chute de la production, l'idée est de s'entendre avec la direction locale pour sauver le site en maintenant l'effectif salarié mobilisé et engagé dans le travail. En retour, la direction valorise cette évolution dans les prises de position en promouvant le rôle des cégétistes dans l'usine. Par exemple, les élus CGT sont associés aux discours des superviseurs sur les chaînes de montage chaque matin ou se voient confier l'animation de commissions de travail sur différents sujets. En retour, ils incitent tout particulièrement les ouvriers de l'usine à remplir positivement l'enquête de satisfaction commandée par le groupe américain propriétaire de l'établissement et, comme la direction, réprouvent les comportements de certains ouvriers qui ne respectent pas les règles de conduite et l'outil de travail dans l'usine. En effet, c'est à cette période, à partir du milieu des années 2000, que les techniques de production dites de lean production (LiNHART, 2010) sont mises en place. Les syndicalistes de la CGT et de FO interrogés expliquent ainsi que l'intensité et la déqualification du travail, la réduction des pauses et l'arrivée de « petits chefs » venus du secteur de l'automobile pour contrôler plus sévèrement les ouvriers provoquent de nombreux conflits avec la direction qui débouchent sur une grève en 2008, juste avant la chute de production en 2009. Certains ouvriers se sentent désormais en décalage avec les pratiques des militants de la CGT, qu'ils jugent trop proches de la direction. Ils décident même de créer une section FO, alors que la CGT restait le seul syndicat de l'entreprise suite à la disparition de la CFDT. Dans cette configuration syndicale nouvelle, le sentiment de discrimination chez les représentants finit par changer de camp, puisque ce sont les élus de ce syndicat minoritaire qui se disent à présent la cible de tentatives de licenciement, de brimades de la part des chefs et qui rencontrent des difficultés pour obtenir les primes et augmentations liées à leurs postes. Pour le délégué syndical de FO et les salariés qui l'ont suivi dans le syndicat, la CGT apparaît, elle, comme l'alliée que la direction cherche à ménager pour apaiser les conflits dans l'établissement.

Ainsi, dans ces espaces de relations et de concurrences entre représentants que constituent les grands établissements, le sentiment de discrimination ne se détermine pas uniquement en fonction des échanges qui se nouent entre les directions et les syndicalistes. Il découle également de la manière dont les représentants comparent les relations qu'ils entretiennent avec leur direction. Pluridimensionnel, le sentiment de discrimination est donc aussi relationnel. Il ne se forge pas uniquement en réponse à ce qui peut être perçu par les syndicalistes comme des stratégies de répression des directions contre le syndicat le plus « puissant » et/ou le plus « combatif ». Les perceptions multiples de la discrimination semblent également se construire en raison de la gestion 
différenciée dont les syndicats font l'objet de la part des directions d'entreprise et du traitement inégal des syndicalistes en fonction de leur organisation d'appartenance. De ce point de vue, la propension différente des représentants syndiqués à se dire discriminés est donc aussi à relier à la manière dont les directions peuvent jouer des divisions syndicales dans leur gestion des relations sociales (BENQUET, 2013).

\section{La maîtrise collective et individuelle du droit : condition d'appropriation du registre de la discrimination?}

Pour comprendre les raisons pour lesquelles le sentiment de discrimination est plus fréquent dans les grands établissements, cerner la variété des motifs de plainte que recouvre ce ressenti ne suffit pas. Sans doute faut-il aussi réfléchir aux mécanismes sociaux qui favorisent l'usage de la notion de discrimination par les représentants dans ce type d'établissements. En effet, tous les représentants qui vivent des rapports conflictuels avec leur direction n'interprètent pas nécessairement cette situation, ni même les traitements défavorables qu'ils estiment subir, en termes de discrimination. On peut faire l'hypothèse que le plus grand degré de formalisation juridique des relations sociales observé dans les grands établissements, parce qu'il contribue à renforcer la familiarisation des syndicalistes au droit, permet également de faciliter leur appropriation de la catégorie « discrimination », qui s'est d'abord diffusée et imposée sur le terrain juridique.

\section{Un usage militant inégal de la catégorie de discrimination}

Dans l'enquête REPONSE, le diplôme influe sur la propension des représentants à se déclarer discriminés : ceux ayant un niveau de diplôme inférieur au baccalauréat ont une probabilité plus faible de se déclarer discriminés (voir tableau 4). Autrement dit, la possession d'un petit capital scolaire et culturel semble favoriser le sentiment d'être freiné dans sa carrière ${ }^{11}$. De plus, si, toutes choses égales par ailleurs, le sexe et l'âge ne jouent pas, les représentants du personnel majoritaires qui sont ouvriers qualifiés ont davantage de chances de se sentir freinés dans leur carrière que les cadres et les techniciens. Ces résultats font écho au travail de V.-A. CHAPPE (2013) qui rappelle que la lutte juridique contre les discriminations a été initiée par des ouvriers qualifiés, l'« aristocratie ouvrière » de l'usine Peugeot à Sochaux, ce qui expliquerait en partie leur volonté collective de voir valoriser des compétences professionnelles déployées

\footnotetext{
11. À titre de comparaison, selon les travaux sociologiques, l'effet du capital scolaire n'est pas univoque sur le sentiment de discrimination. Certaines enquêtes sur les discriminations raciales pointent le fait que ce sont les moins dotés qui se sentent les plus discriminés (PRIMON, 2011); d'autres indiquent au contraire que ceux-ci perçoivent moins ce type de discrimination qu'ils vivent comme une injustice parmi d'autres (DuBET et al., 2013). On peut faire l'hypothèse que c'est l'interaction du capital culturel avec des situations vécues et d'autres types de ressources (militantes par exemple) qui joue sur cette perception.
} 
à l'usine qu'ils estimaient non reconnues dans un contexte de reflux syndical et de déclin des mobilisations (CHAPPE, 2013). À partir de nos monographies, il est possible de prolonger cette analyse : les trajectoires sociales des représentants du personnel se combineraient avec leur inégale socialisation au droit pour expliquer leurs dispositions inégales à se saisir de la notion de discrimination.

Les représentants des trois organisations syndicales implantées dans l'établissement Bus se plaignent de relations conflictuelles avec une direction qui leur serait très hostile. Ceci concerne autant les délégués de l'Union Solidaire des Transport (UST), syndicat majoritaire depuis 2006, que le délégué central de la Fédération nationale des chauffeurs routiers (FNCR, syndicat indépendant), deuxième syndicat représentatif, et que le représentant de la section syndicale de la Confédération française des travailleurs chrétiens (CFTC), non représentative. La comparaison des discours tenus par M. Nyobe, délégué syndical central du syndicat UST et de M. Poniatowski, représentant de la section CFTC, est particulièrement instructive. L'entretien collectif conduit en présence de ces deux militants met en évidence deux manières distinctes d'interpréter les conflits qui les opposent à leur direction dans l'exercice de leur mandat. Alors que M. Poniatowski utilise les termes de discrimination et de harcèlement et fait appel aux ressources du droit pour tenter de régler ces litiges, M. Nyobe, lui, ne fait pas référence à ces registres d'action et de discours, et privilégie un mode de confrontation plus direct avec sa hiérarchie :

M. Nyobe : « Dans les réunions que je fais ici, je pose des heures de délégation parce que je ne vais pas travailler gratuitement et mon supérieur juge lui-même si j'ai droit ou pas droit. [...] J'ai été lui demander parce qu'il y a toujours des calculs un peu... Je lui demande : "Il me reste combien d'heures ?" Il m'a répondu : "Va te faire foutre avec tes heures de délégation!' Il m'a dit ça. Moi, j'ai voulu le taper, j'ai levé ma main et j'ai tapé sur la table. Il y avait monsieur le chef d'équipe qui était à l'autre bout du couloir, il se met entre nous : "Non, ne le tape pas ! " [...] Et ils ont fait une combine, un faux témoignage comme quoi je l'avais tapé. »

M. Poniatowski : « Moi, j’ai dû recevoir en cinq ans soixante lettres recommandées, des avertissements, que j'ai déjoués à chaque fois. Quand je dis "déjoués", c'est vice de procédure. Moi, je suis harcelé quotidiennement, constamment ; je réussis à chaque fois, plus malin qu'eux, je connais aussi un peu le droit. »

(Entretien réalisé le 14 décembre 2012)

La mise en perspective des profils professionnels de ces deux représentants et de leurs rapports différents à l'action syndicale permet d'éclairer cette tendance différentielle à se référer à la notion de discrimination et aux modes d'action juridique permettant d'en obtenir réparation. De nationalité malienne et âgé d'une cinquantaine d'années, M. Nyobe vit en France depuis une dizaine d'années. Il n'est pas diplômé et éprouve de grandes difficultés à l'écrit. Lors des négociations, il est régulièrement contraint de signer des documents dont, aux dires de la DRH, il ne comprend pas le contenu. Sa maîtrise des procédures juridiques organisant les relations professionnelles est elle-même approximative. Il commet par exemple des erreurs récurrentes 
lors du dépôt de préavis de grèves, aboutissant à leur invalidation. Mal à l'aise avec l'écrit et le langage formel et juridique des négociations, M. Nyobe a recours à des modes de confrontation directe avec la direction : intimidations physiques à l'égard de l'encadrement, grèves de longue durée, occupations des locaux de l'établissement, blocage d'axes routiers, etc. Son rapport distant au droit aide à comprendre qu'il privilégie d'autres ressources pour s'imposer face à sa direction. Dans le contexte de son entreprise, M. Nyobe apparaît d'autant moins enclin à se vivre comme un syndicaliste discriminé que sa liste arrive en tête des élections professionnelles, que son établissement n'offre aucune véritable possibilité de promotion professionnelle pour ses chauffeurs et que la confrontation avec sa direction s'inscrit dans la continuité de conflits eux-mêmes récurrents entre les salariés et le management. Dans ces conditions, il ne peut guère percevoir son engagement syndical comme un frein à sa carrière ou se sentir victime d'un traitement particulier de la part de sa direction.

Les conditions de l'engagement syndical de M. Poniatowski se distinguent de deux points de vue de celles de M. Nyobe. Détenteur d'un CAP, c'est un ancien chef d'équipe ayant longtemps travaillé dans l'industrie avant d'être licencié. Il vit son nouvel emploi comme un déclassement professionnel et décide de fonder une section CFTC afin de se consacrer à une activité qu'il pense utile et plus intéressante. Pendant plusieurs années, il suit les formations juridiques organisées par la CFTC et étudie minutieusement tous les règlements que l'entreprise est censée respecter. Ayant tout d'abord l'intention d'établir de bonnes relations avec la direction et avec les autres syndicats (UST et FNCR), il est très vite déçu par le peu de cas qui est fait de son opinion pendant les négociations, alors même qu'il se sent bien plus compétent que les autres délégués syndicaux. Cette situation l'incite à contester davantage les pratiques de la direction, en rédigeant notamment des tracts dans lesquels il dénonce les illégalités qu'elle commet ainsi que ses connivences avec certains syndicalistes. Il affirme avoir fait également appel à l'inspection du travail en raison du non-respect des temps de pause par la direction, avoir engagé une procédure auprès du tribunal administratif de la Sécurité sociale et avoir envoyé des courriers aux organismes de certification, preuves à l'appui, concernant le non-respect de normes de qualité. Il est convaincu que ces actions représentent une menace sérieuse pour la direction, qui cherche pour cette raison, d'une part à les empêcher, d'autre part à le licencier. Faisant référence à des textes de lois et à des règlements précis, il dénonce dans ses tracts la discrimination syndicale dont il s'estime victime : entraves à son action (retrait de sa boîte aux lettres syndicale par exemple) ; tracasseries administratives diverses (invention de faux retards, notamment) ; provocations racistes de la part de $l^{\prime}$ encadrement ${ }^{12}$. M. Poniatowski mène cependant le plus souvent seul ces actions juridiques. Il se sent peu soutenu par les autres syndicalistes dans les démarches qu'il entreprend, et regrette que les salariés ne mesurent pas l'efficacité de ses initiatives. Cet isolement se traduit en 2009 à la fois par la perte, pour la CFTC, de son statut de

12. Dans une lettre ouverte à la direction, M. Poniatowski dénonce ainsi des propos ayant, d'après lui, été tenus à son égard par un contremaître : «Aujourd'hui je suis bien remonté et j'ai envie de casser du Polonais. » 
syndicat représentatif et pour lui, par conséquent, par celle de son mandat de délégué syndical. La situation le renforce dans son sentiment d'être marginalisé et l'entretient dans l'idée que ses qualités (professionnelles et militantes) ne sont pas reconnues à leur juste valeur au sein de Bus.

On voit bien ici comment les effets de la socialisation au droit se combinent avec ceux de la position dans le champ des relations professionnelles et des attentes en termes de carrière professionnelle dans l'émergence du sentiment d'être pénalisé par son activité militante et dans l'usage du cadre interprétatif juridique de la discrimination pour l'exprimer. L'appropriation du discours juridique de la discrimination ne dépend cependant pas uniquement de trajectoires scolaires et professionnelles individuelles. Elle varie aussi en fonction des stratégies collectives développées et des savoir-faire accumulés à l'intérieur des collectifs militants dans lesquels s'inscrit l'action des syndicalistes.

\section{Les conditions d'une judiciarisation croissante de la lutte pour la reconnaissance des droits syndicaux}

Pour M. Henri, l'adhésion à la CGT de Transformateur a été l'occasion de se former au droit des institutions représentatives du personnel. Il a ainsi intégré l'idée, au contact des plus anciens militants de son syndicat et des autres syndicats de la métallurgie qu'il fréquente dans son union locale, que la répression anti-syndicale est une dimension structurante des relations professionnelles du secteur, dimension contre laquelle il est nécessaire d'apprendre à se défendre :

«Les gars, au syndicat, ils m' avaient averti : on cherche toujours quelque chose pour nous saquer. Moi, je le vois bien avec mon supérieur. Il me dit que je suis un très bon élément. Mais dès que je demande quelque chose, dès qu'il y a une prime, c'est non. Après, moi je sais qu'il y a la loi, le Code du travail, la convention... et on est obligé de se battre avec tous ces éléments-là. »

(Entretien réalisé le 22 mai 2013)

Dans l'établissement Engins, la dénonciation par les représentants des traitements discriminatoires dont ils s'estiment victimes s'inscrit là aussi dans le prolongement d'une lutte plus ancienne et collective contre les discriminations syndicales : M. Brénot, ancien secrétaire de la CGT, avait constitué avec d'autres militants un « dossier » pour obtenir réparation des pénalités qu'ils considéraient avoir subies dans leurs rémunérations et dans l'évolution de leur carrière professionnelle. En dépit de l'accord trouvé avec la direction pour retirer cette plainte, M. Moreira, élu CGT au comité d'entreprise, affirme que la direction attribue encore des salaires ou des augmentations salariales moins élevés aux syndicalistes, au motif qu'ils bénéficient d'heures de délégations et qu'ils travaillent donc moins que les autres salariés. Il admet d'ailleurs prévenir ses jeunes collègues qui veulent adhérer à la CGT des risques auxquels ils s'exposent. Les militants du syndicat ne s'y résignent cependant pas. Soucieux de maintenir une base militante - le syndicat revendique une cinquantaine d'adhérents -, les cégétistes ont 
plusieurs fois menacé la direction d'engager des actions juridiques pour la contraindre à revaloriser ou du moins à ne pas discriminer les syndicalistes de l'usine. Le travail de conscientisation et de politisation de la question des discriminations favorise en retour les perceptions individuelles, et sûrement des formes de relecture de leurs trajectoires par les syndicalistes, en termes de discrimination, processus qu'on retrouve également concernant la perception des discriminations de genre ou ethniques (POIRET, 2010).

Les stratégies de mobilisation du droit peuvent être mises en œuvre grâce aux compétences juridiques acquises par ces syndicalistes auprès des plus anciens militants, ou à l'occasion de formations syndicales. Ils bénéficient en outre du soutien de l'union locale et départementale de la CGT qui, située dans un bassin industriel important, a une taille critique et des ressources financières suffisantes pour systématiser ce type de procédure ou former ses délégués à la méthode de François Clerc (CHAPPE, 2011). Les deux établissements industriels étudiés mettent en évidence que, dans ce type d'entreprises, si le capital scolaire des militants ouvriers reste modeste, l'histoire des luttes syndicales et la présence d'une équipe militante solidement ancrée rendent possibles des apprentissages collectifs qui facilitent la familiarisation des militants syndicaux à l'usage du mode d'action juridique, et favorisent la diffusion de la catégorie juridique de discrimination comme cadre de référence et comme instrument de lutte pour dénoncer ce qui est perçu comme des stratégies patronales anti-syndicales.

Ce processus de judiciarisation de la lutte pour la reconnaissance des droits des militants syndicaux a de surcroît été encouragé ces dernières années par l'évolution des stratégies syndicales en matière de lutte contre la répression patronale. En valorisant le caractère héroïque de l'action syndicale, la CGT a, en particulier, longtemps eu tendance à considérer la répression patronale comme le prix à payer de l'engagement dans la « lutte des classes ». Dans un contexte d'affaiblissement militant important, la centrale syndicale a, depuis la fin des années 1990, fait au contraire de la lutte juridique contre les discriminations de ses militants un axe décisif de son travail de formation de ses adhérents pour faciliter le redéploiement de l'organisation (HATZFELD, 2014 et dans ce numéro).

Que, dans l'enquête REPONSE, les déclarations de pénalisation des carrières du fait de l'activité de représentant du personnel soient plus élevées dans les établissements de grande taille n'implique donc pas que les faits de discrimination syndicale soient nécessairement plus fréquents dans ces établissements que dans d'autres. Ce résultat n'entre pas non plus en contradiction avec la codification juridique croissante des relations professionnelles dans ces organisations. Au contraire, ce processus contribue à une judiciarisation des conflits du travail (PÉLISSE, 2009), et crée précisément des conditions plus propices pour que les litiges qui opposent les syndicalistes à leurs employeurs dans l'application de leurs droits soient plus souvent formulés en termes de discrimination. 
La combinaison des données statistiques et des enquêtes monographiques permet de faire ressortir la diversité des formes de discrimination ressenties par les représentants du personnel, et le caractère tout à la fois relationnel et contingent de ce sentiment. À côté des acquis produits par les récents travaux s'étant attelés à objectiver statistiquement les pratiques de discrimination anti-syndicale (cf. par exemple Bourdieu, Breda, ou Guiomard, Meftah, dans ce numéro), l'ambition de cet article était de montrer ce qu'une approche compréhensive du phénomène de discrimination syndicale apporte à l'analyse de la variété des ressorts et des contours du sentiment de discrimination exprimé par certains représentants du personnel syndiqués.

Il ressort en premier lieu de l'analyse statistique que les facteurs qui jouent sur le sentiment de discrimination sont similaires à ceux qui déterminent les conditions dans lesquelles les représentants du personnel subissent des pénalités salariales. Ainsi, l'appartenance syndicale, l'intensité des interactions entre représentants du personnel et dirigeants, de même que l'existence de relations sociales conflictuelles tendent à favoriser l'expression de ce sentiment. On peut dès lors comprendre qu'il soit plus prononcé dans les grands établissements, là où les relations professionnelles sont plus institutionnalisées, puisque c'est aussi dans ces établissements que les confrontations et les conflits entre directions et syndicalistes sont plus fréquents. Dans ces établissements dotés d'une présence syndicale souvent pluraliste, la question des droits syndicaux et de la reconnaissance de l'action des syndicalistes se constitue plus fréquemment en enjeu de luttes entre les acteurs en présence. De ce point de vue, à la suite d'autres travaux (CHAPPE, 2013), nous constatons que la discrimination syndicale a d'autant plus de chances d'être ressentie et appropriée comme un enjeu de lutte que les syndicalistes disposent des ressources militantes individuelles et collectives nécessaires pour appréhender et mobiliser les conventions juridiques que recouvre cette catégorie. Dans un contexte où les organisations syndicales sensibilisent et forment davantage leurs adhérents aux outils du droit, c'est sans doute l'un des éléments qui explique le maintien d'un niveau élevé de sentiment de discrimination parmi les représentants du personnel des grands établissements, en dépit d'une légalisation apparente des politiques managériales de gestion des relations sociales.

Mais à travers nos enquêtes monographiques, nous avons également mis en évidence le caractère relationnel du sentiment de discrimination exprimé par les représentants du personnel. De fait, ce sentiment ne se construit pas nécessairement en référence à des normes juridiques, mais se construit aussi (et surtout) en fonction de la dynamique des interactions qui se nouent entre les différents acteurs de l'entreprise. La perception de la discrimination par les représentants du personnel varie en fonction du caractère plus ou moins conflictuel des relations entre représentants du personnel et dirigeants. Et elle s'articule, pour les syndicalistes interrogés, à des formes plus collectives d'entraves à leur activité de représentant. Si l'action publique de lutte contre les discriminations syndicales se focalise sur la mise en place de dispositifs légaux de suivi des parcours et des carrières des représentants du personnel, il ressort de notre 
enquête que ces dispositifs n'épuisent pas la problématique des discriminations et des formes d'entrave auxquelles les représentants du personnel considèrent faire face.

De même, les enquêtes monographiques suggèrent que le sentiment de discrimination ne se nourrit pas seulement du rapport aux directions d'entreprise : il se construit aussi par le jeu des comparaisons et concurrences entre syndicalistes dans le type de relations qu'ils entretiennent avec leur direction. Ainsi, les positions relatives occupées par les représentants dans le champ syndical des établissements, leurs manières différentes d'investir leur rôle et les modalités de leur socialisation militante jouent sûrement au moins autant que l'affiliation syndicale dans l'émergence d'un tel sentiment. La cause de la discrimination syndicale ne s'ancre donc pas seulement dans les supports conventionnels et matériels dont peuvent bénéficier les syndicalistes, mais s'ancre aussi en lien avec l'habitus des militants, leur positionnement dans l'espace syndical et les rapports de force dans le champ économique.

La perception de la discrimination syndicale se construit donc au croisement de quatre types de rapports sociaux : le rapport aux autres salariés, qui explique que le sentiment de discrimination puisse être plus fréquent dans les grands établissements, où les représentants du personnel syndiqués peuvent plus aisément se comparer aux salariés non syndiqués ; le rapport aux directions d'entreprise, marqué par le sentiment d'être plus ou moins désavantagés selon l'état des relations et des rapports de force entre représentants du personnel et dirigeants ; le rapport au droit, et plus spécifiquement à la notion de discrimination, qui détermine en partie la capacité des syndicalistes à qualifier leur situation de cette manière et à initier des procédures contre ce dont ils s'estiment victimes ; le rapport des syndicalistes entre eux enfin, qui, par le biais des pratiques distinctives entre syndicats et/ou syndicalistes selon leurs positions et leurs trajectoires militantes, favorise plus ou moins le sentiment de discrimination. C'est l'articulation de ces différents rapports sociaux qui gagnerait à être questionnée de manière plus systématique dans de prochaines enquêtes statistiques et monographiques.

\section{BibLIOGRAPHIE}

Algava É., BÈQue M. (2006), « Perception et vécu des comportements intolérants. Une analyse du module "Relations avec les autres" de l'enquête Histoire de vie ", Économie et Statistique, no 393-394, pp. 115-150.

BENQUET M. (2013), « Le circuit de secours syndical. Quand représentants patronaux et syndicaux cogèrent les conflits professionnels », Agone, $\mathrm{n}^{\circ}$ 50, pp. 135-157.

Béroud S., Denis J.-M., Desage G., Giraud B., Pélisse J. (2008), La Lutte continue ? Les conflits du travail dans la France contemporaine, Bellecombe-en-Bauges, Éditions du Croquant.

BouRDIEU J., BREDA T. (2016), « Des délégués syndicaux sous-payés : une situation de discrimination stratégique ? Une analyse économétrique à partir de l'enquête REPONSE de 2010 », Travail et Emploi, $\mathrm{n}^{\circ} 145$, pp. 31-58. 
Bourdieu J., BredA T. (2015), Les Employeurs face aux représentants du personnel : une situation de discrimination stratégique, Rapport final pour la Dares.

BREDA T. (2014), « Les délégués syndicaux sont-ils discriminés ? », Revue économique, vol. 65, $\mathrm{n}^{\mathrm{o}} 6$, pp. 841-880.

Brinbaum Y., GuÉGNARD C. (2012), «Le sentiment de discrimination des descendants d'immigrés : reflet d'une orientation contrariée et d'un chômage persistant », Agora débats/ jeunesses, $\mathrm{n}^{\mathrm{o}}$ 61, pp. 7-20.

ChAppe V.-A. (2011), « La preuve par la comparaison : méthode des panels et droit de la nondiscrimination », Sociologies pratiques, ${ }^{\circ}$ 23, pp. 45-55.

CHAPPE V.-A (2013), « Dénoncer en justice les discriminations syndicales : contribution à une sociologie des appuis conventionnels de l'action judiciaire », Sociologie du travail, vol. 55, $\mathrm{n}^{\mathrm{o}} 3$, pp. 302-321.

Corouge C., Pialoux M. (2011), Résister à la chaîne. Dialogue entre un ouvrier de Peugeot et un sociologue, Marseille, Agone.

Denis J.-M. (2009), « Dans le nettoyage, c'est le chantier qui part en grève ! », in Béroud S., Bouffartigue P. (dir.), Quand le Travail se précarise, quelles résistances collectives?, Paris, La Dispute, pp. 99-116.

Dubet F., Cousin O., MacéÉ., RuI S. (2013), Pourquoi moi ? L'expérience des discriminations, Paris, Seuil.

ECKert H., Primon J.-L. (2011), « Introduction. Enquêter sur le vécu de la discrimination », Agora débats/jeunesses, $\mathrm{n}^{\circ}$ 57, pp. 53-61.

Gantois M. (2014), « La “démocratie d'entreprise” en actes. Ce que l'observation d'une négociation révèle des relations professionnelles "ordinaires" dans une PME », Politiques de communication, $\mathrm{n}^{\circ} 2$, pp. 79-112.

Giraud B., Penissat E., PÉlisse J. (2014), Gérer, négocier, résister. Une approche dynamique des politiques patronales, des négociations et des conflits au travail (des années 2000 à aujourd'hui), Rapport de recherche pour la Dares. Disponible en ligne : https://halshs.archivesouvertes.fr/halshs-01083780/document ; consulté le 18 juillet 2016.

GIRAUD B. (2013), «Derrière la vitrine du "dialogue social” : les techniques managériales de domestication des conflits du travail », Agone, $\mathrm{n}^{\circ} 50$, pp. 33-63

GIRAUD B (2007), «Le syndicalisme saisi par le management. Les conditions de production d'une expertise managériale de l'action syndicale au prisme de ses investissements diversifiés », Politix, $\mathrm{n}^{\mathrm{o}} 79$, pp. 125-147.

Groux G. (1998), Vers un Renouveau du conflit social ?, Paris, Bayard.

HATZFELD N. (2014), « Lutte contre la discrimination syndicale à Peugeot-Sochaux : combats judiciaires et mutations syndicales (1995-2000) », in Narritsens A., Pigenet M. (dir.), Pratiques syndicales du droit : France, $X X^{e}-X X I^{e}$ siècles, Rennes, Presses universitaires de Rennes, pp. 255-264 
HATZFELD N. (2016), « Une lutte syndicale exemplaire chez Peugeot-Sochaux (1995-2000). La remise en cause d'un système discriminatoire », Travail et Emploi, n 145, pp. 173-196.

Linhart D. (2010), La Modernisation des entreprises, 3 e éd., Paris, La Découverte.

PÉLISSE J. (2009), « Judiciarisation ou juridicisation? Usages et réappropriations du droit dans les conflits du travail », Politix, n⿳0 86, pp. 73-86.

Piotet F. (2009), La CGT et la recomposition syndicale, Paris, Presses universitaires de France.

PAK M., Pignoni M.-T. (2014), « Les représentants du personnel : quelles ressources pour quelles actions ? », Dares Analyses, $n^{\circ} 84$.

Pignoni M.-T., RAYNAUd É. (2013), « Les relations professionnelles au début des années 2010 : entre changements institutionnels, crise et évolutions sectorielles », Dares Analyses, $\mathrm{n}^{\circ} 26$.

PoIRET C. (2010), « Pour une approche processuelle des discriminations : entendre la parole minoritaire », Regards sociologiques, $\mathrm{n}^{\circ}$ 39, pp. 5-20.

PRIMON J.-L. (2011), « La perception des discriminations au filtre des enquêtes statistiques », Agora débats/jeunesse, n 57, pp. 121-134.

OBSERVATOIRE DE LA DISCRIMINATION ET DE LA RÉPRESSION SYNDICALES (2014), De la discrimination individuelle à l'action collective : propositions pour garantir le respect des droits syndicaux et salariés, rapport 2014.

Silberman R., FouRnier I. (2006), « Les secondes générations sur le marché du travail en France : une pénalité ethnique ancrée dans le temps. Contribution à la théorie de l'assimilation segmentée. Contribution à la théorie de l'assimilation segmentée », Revue française de sociologie, vol. 47, $\mathrm{n}^{\mathrm{o}} 2$, pp. 243-292.

Schwalbe M., Godwin S., Holden D., Schrock D., Thompson S., Wolkomir M. (2000), "Generic Processes in the Reproduction of Inequality: An Interactionist Analysis", Social Forces, vol. 79, n 2, pp. 419-452.

TILly C. (1998), Durable Inequality, Berkeley (Californie), University of California Press.

WiLlemeZ L. (2012), «Quand le syndicalisme n’est pas une mince affaire... », Savoir/Agir, $\mathrm{n}^{\mathrm{o}} 21$, pp. 79-84. 